\title{
Multiscale Morphological Visualization of a Semi-crystalline Polymer by a Polymorphic AIE Marker
}

Michidmaa Khorloo, yanhua Cheng, Haoke Zhang, Ming Chen, Herman H.-Y. Sung, lan Duncan Williams, Jacky W. Y. Lam, Ben Zhong Tang

Submitted date: 05/07/2019 - Posted date: 05/07/2019

Licence: CC BY-NC-ND 4.0

Citation information: Khorloo, Michidmaa; Cheng, yanhua; Zhang, Haoke; Chen, Ming; Sung, Herman H.-Y.; Williams, Ian Duncan; et al. (2019): Multiscale Morphological Visualization of a Semi-crystalline Polymer by a Polymorphic AIE Marker. ChemRxiv. Preprint.

Direct spatial mapping of polymer morphologies remains challenging owing to poor imaging contrast across different microstructures. Now this conundrum is addressed by a polymorphic molecule with aggregation-induced emission (AIE), capable of selectively lighting up amorphous and crystalline phases with distinct color. The polymorphic behaviors of the AIE molecule embedded within polymer network is dependent on the size of nano-confinement: a thermodynamically stable polymorph of the AIE molecule with green emission is stabilized in amorphous phase, while a metastable polymorph with yellow emission is confined in crystalline phase. The information on polymer crystalline and amorphous phases is transformed into distinct fluorescence colors, allowing a single AIE molecule as a fluorescent marker for multiscale visualization of polymer morphologies in terms of amorphous and crystalline phases distribution, quantitative polymer crystallinity measurement, and spatial morphological arrangement. The proposed strategy provides a correlation between microscopic morphologies and macroscopic optical signals, opens an avenue of fluorescence imaging in materials science.

File list (2)

MS_MKCYH.pdf (1.25 MiB)

view on ChemRxiv • download file

SI_MKCYH.pdf (1.83 MiB)

view on ChemRxiv - download file 


\section{Multiscale Morphological Visualization of a Semi-}

\section{crystalline Polymer by a Polymorphic AIE Marker}

Michidmaa Khorloo, ${ }^{\dagger, \perp}$ Yanhua Cheng, ${ }^{*,+, \perp}$ Haoke Zhang,,$^{\dagger}$ Ming Chen,$^{\dagger}$ Herman H. Y. Sung, ${ }^{\dagger}$ Ian D. Williams, ${ }^{\dagger}$ Jacky W. Y. Lam ${ }^{\dagger}$ and Ben Zhong Tang $*, \dagger,,, \|$

†Department of Chemistry, the Hong Kong Branch of Chinese National Engineering Research Center for Tissue Restoration and Reconstruction, Institute for Advanced Study and Department of Chemical and Biological Engineering, The Hong Kong University of Science and Technology, Clear Water Bay, Kowloon, Hong Kong, China. E-mail: tangbenz@ust.hk ${ }^{\ddagger}$ State Key Laboratory for Modification of Chemical Fibers and Polymer Materials, College of Materials Science and Engineering, Donghua University, Shanghai, 201620, China. E-mail: cyh@dhu.edu.cn

${ }^{\S}$ HKUST-Shenzhen Research Institute, No. 9 Yuexing $1^{\text {st }}$ RD, South Area, Hi-tech Park, Nanshan, Shenzhen 518057, China

"Center for Aggregation-Induced Emission, SCUT-HKUST Joint Research Institute, State Key Laboratory of Luminescent Materials and Devices, South China University of Technology, Guangzhou 510640, China

\section{KEYWORDS:}

morphological visualization, polymorphism, fluorescent marker, semi-crystalline polymer, aggregation-induced emission 


\section{ABSTRACT}

Direct spatial mapping of polymer morphologies remains challenging owing to poor imaging contrast across different microstructures. Now this conundrum is addressed by a polymorphic molecule with aggregation-induced emission (AIE), capable of selectively lighting up amorphous and crystalline phases with distinct color. The polymorphic behaviors of the AIE molecule embedded within polymer network is dependent on the size of nano-confinement: a thermodynamically stable polymorph of the AIE molecule with green emission is stabilized in amorphous phase, while a metastable polymorph with yellow emission is confined in crystalline phase. The information on polymer crystalline and amorphous phases is transformed into distinct fluorescence colors, allowing a single AIE molecule as a fluorescent marker for multiscale visualization of polymer morphologies in terms of amorphous and crystalline phases distribution, quantitative polymer crystallinity measurement, and spatial morphological arrangement. The proposed strategy provides a correlation between microscopic morphologies and macroscopic optical signals, opens an avenue of fluorescence imaging in materials science. 
Morphology is an indispensable aspect of nature materials, present in exquisite microstructures, accounting for the mechanical properties and biological functions of living systems. Such phenomenon can be illustrated by adhesive properties of gecko foot-hair, ${ }^{1}$ toughness of nacre ${ }^{2}$ and super-hydrophobicity of lotus leaf. ${ }^{3}$ This principle has inspired researchers to design and fabricate high-performance synthetic materials with versatile functions through morphology engineering at the microscale length. ${ }^{4-6}$ Among them, semicrystalline polymers occupy a very large proportion, ${ }^{7}$ covering more than two-thirds of the global applications of polymer materials. Their intrinsic capability to arrange macromolecular segments into three-dimensional (3D) ordered crystal structures with different morphologies determine the final functional properties. ${ }^{89}$ Examples include the crystalline size and distribution control of semiconducting polymer to improve the performance of solar cells; ${ }^{10}$ the crystallinity variation in conductive polymer to facilitate alcohol vapor sensing; ${ }^{11}$ the morphology transition from spherulitic to shish-kebab to afford stiffness improvement. ${ }^{12}$ Therefore, the direct morphological observation of semi-crystalline polymer materials in both two-dimensional (2D) and 3D form is highly important from conceptual and practical perspectives. However, direct observation of morphologies using current electron microscopy is hampered due to the low specificity of polymer structure to electron bombardment. ${ }^{13}$

Fluorescence imaging is an ideal method that offers a simple, accurate and easy-readable way for spatial morphology visualization in soft materials. ${ }^{14,15}$ Such technique provides information that relates not only to the outer surface of targeted materials, but also interior morphology within the materials body facilitated by confocal fluorescence microscopy (CFM). ${ }^{16,17}$ Generally, fluorescent dyes or proteins serve as "built-in" sensors in the targeted micro-environment to extract information about their molecular process or structures. ${ }^{15,18}$ However, few demonstrations have been realized in polymer matrix due to the lack of effective and versatile fluorophores in the condensed state. ${ }^{19}$ The development of new fluorescent 
systems with sensitive optical response to the polymer microenvironment would facilitate mapping the materials morphologies.

A molecular system characterized with aggregation-induced emission (AIE) is well suited for such purposes. AIE describes a general phenomenon that molecules are non-luminescent in solution but give intense emission when aggregated. ${ }^{19}$ The restriction of intramolecular motions (RIM) has been recognized as the mechanism of the AIE phenomenon, which prevents the dissipation of excited-state energy through nonradiative decay channels. ${ }^{20}$ According to the RIM mechanism, structural rigidification of molecules in high viscosity/rigid media and low temperature result in enhanced emission. ${ }^{21,22}$ Based on these characteristics, several AIE systems have been successfully exploited for examining materials morphologies, ${ }^{19}$ including their hard-soft phase distribution, ${ }^{23,24}$ inorganic-organic component characterization, ${ }^{25}$ polymer viscosity monitoring, ${ }^{26}$ self-assembly evolution, ${ }^{27}$ and polymer gelation process ${ }^{28}$ Nonetheless, these systems normally exploited significant variations in the physical properties of materials, which originate from their different chemical compositions. In contrast, labeling different microstructures of a material with same elements requires highly sensitive fluorescent system.

One possible strategy for designing morphological visualizers involves the incorporation of donor-acceptor (D-A) structures into AIE molecules to develop AIE-active fluorescent molecular rotors with distinct color. ${ }^{29}$ Their twisted 3D molecular conformation and weak intermolecular interactions afford them to show multiple structural transformability, ${ }^{30-32}$ generating variable metastable states with high-contrast emission properties that are sensitive to the local microenvironment. ${ }^{32,33}$ In addition, our previous study indicate that the metastable state of the AIE molecules could be transformed into thermodynamically stable one when glassified in polymer matrix. ${ }^{34}$ These features uncover the possibility of AIE molecules to show exquisite labeling specificity to polymer environment using metastable states. As a result, a 
foundation could be established between microscopic morphologies and macroscopic optical signals.

Herein, we report a strategy of using a single AIE molecular marker for 3D morphological visualization of poly(L-lactide) (PLLA). These AIE markers are detected as differences in the crystalline polymorphic states when encoded in polymeric amorphous and crystalline phases. An amphiphilic D-A based AIE molecule (denoted as TPE-EP) was used by attaching a pyridinium salt unit to tetraphenylethene (TPE) group via a double bond (Figure 1a), yielding segregated solid structures by separating its hydrophilic and hydrophobic units. The fluorescence of TPE-EP in the aggregate state depends on the polymorphic forms: crystalline forms $\mathbf{G}$ (thermodynamic stable state), $\mathbf{Y}$ (metastable state) and $\mathbf{O}$ (metastable state), which exhibit green, yellow and orange emission, respectively. The amphiphilic structure forces the molecules to form segregated nanocrystals in hydrophobic PLLA: in amorphous PLLA, molecular aggregates in polymorph $\mathbf{G}$ are stabilized in loose network; in crystalline PLLA, molecular aggregates in polymorph $\mathbf{Y}$ are confined in between lamellas. Such phenomenon allows TPE-EP to operate as a fluorescent marker for multiscale visualization of polymer morphologies, including amorphous and crystalline phases distribution, quantitative polymer crystallinity measurement, and spatial morphological arrangement. The AIE molecules with suitable polymorphic characteristics are expected to have broad applications in diverse polymers, facilitating both mechanistic studies of morphological formation and evolution as well as the development of fluorescent materials with tunable emission. 


\section{RESULTS AND DISCUSSION}
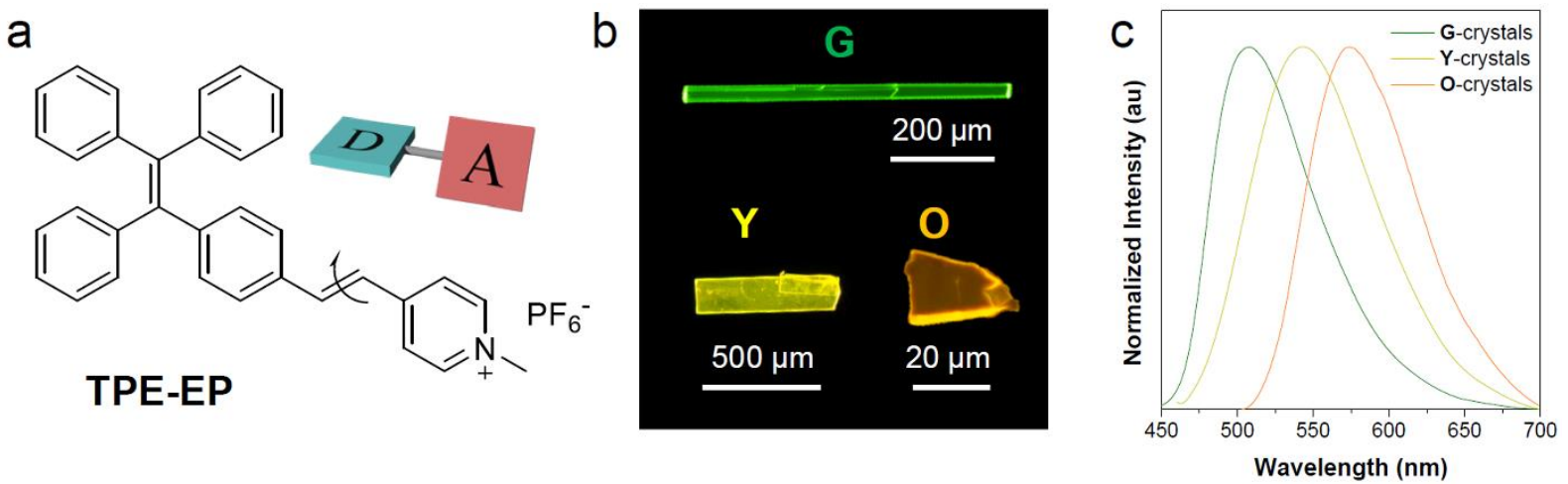

Figure 1. Structural and optical properties of TPE-EP. (a) D-A based AIE molecule. (b) Fluorescent photographs of polymorphic G-, Y-, and O-crystals as well as their (c) corresponding emission spectra. Excitation wavelength: $405 \mathrm{~nm}$.

Molecular information and polymorphic properties. In this study, TPE-EP as a "built-in" marker was applied for simultaneous 2D/3D morphological visualization of semi-crystalline PLLA. TPE-EP consists of three subunits: a hydrophobic TPE as electron donor unit, a hydrophilic pyridinium salt group as electron acceptor unit, and double bond as a spacer unit (Figure 1a). By controlling the precipitation conditions, three crystalline forms $(\mathbf{G}, \mathbf{Y}$ and $\mathbf{O})$ can be produced. Figure 1b shows the photos of G-, Y-, and $\mathbf{O}$-crystals taken under fluorescent microscope. Green photoluminescence was observed from G-crystals under UV excitation (405 $\mathrm{nm})$ with emission maximum at $507 \mathrm{~nm}$, quantum yield $\left(\Phi_{\mathrm{F}}\right)$ of 0.21 and life time $(\tau)$ of $2.1 \mathrm{~ns}$ (Table S1; Figure 1c, green solid line). The crystal structure of $\mathbf{G}$ was determined by singlecrystal X-ray diffraction (XRD). G-crystals were found to be monoclinic (P21/n, $a=11.2525(2)$ $\AA, b=9.21940(13) \AA, c=59.1397(9) \AA, \alpha=90^{\circ}, \beta=93.8053(14)^{\circ}, \gamma=90^{\circ}$ at $100.03 \mathrm{~K}, \mathrm{Z}=4$, goodness of fit $(\mathrm{GOF})=1.036$, calculated density: $1.384 \mathrm{~g} \mathrm{~cm}^{-3}$, Table S2). The polymorphic Y-crystals (Figure 1b) were produced by slow evaporation of a tetrahydrofuran/hexane mixture of TPE-EP, and showed a yellow emission $\left(\lambda_{\mathrm{em}}=543 \mathrm{~nm}\right)$ under UV irradiation (405 nm, Figure 
1c, yellow solid line). Y-crystals were found to exhibit a $\Phi_{\mathrm{F}}$ of 0.32 (Table S1, Supporting Information). The crystal structure of $\mathrm{Y}$ was also monoclinic with a space group of $\mathrm{P} 21 / \mathrm{c}(a=$ 24.8939(7) $\AA, b=9.1783(2) \AA, c=12.5794(3) \AA, \alpha=90^{\circ}, \beta=98.168(3)^{\circ}, \gamma=90^{\circ}$ at $100.01 \mathrm{~K}$, $\mathrm{Z}=4$, goodness of fit $(\mathrm{GOF})=1.019$, calculated density: $1.390 \mathrm{~g} \mathrm{~cm}^{-3}$, Table S2). O-crystals showed orange emission $\left(\lambda_{\mathrm{em}}=575 \mathrm{~nm}\right)$ but it is too brittle to be studied by single crystal XRD. A thermal phase transformation from $\mathrm{Y}$ to $\mathrm{G}$ was revealed at elevated temperature of $116{ }^{\circ} \mathrm{C}$ (Figure S1). No reverse phase was observed when the sample was cooled. Y-crystals were only obtained by re-dissolution and recrystallization process. Similar phenomenon was also observed in $\mathbf{O}$-crystals (Figure S1). The thermal analyses suggest that $\mathbf{G}$ is a thermodynamically favorable form, while $\mathbf{Y}$ - and $\mathbf{O}$-crystals are metastable (Figure S2). ${ }^{35}$

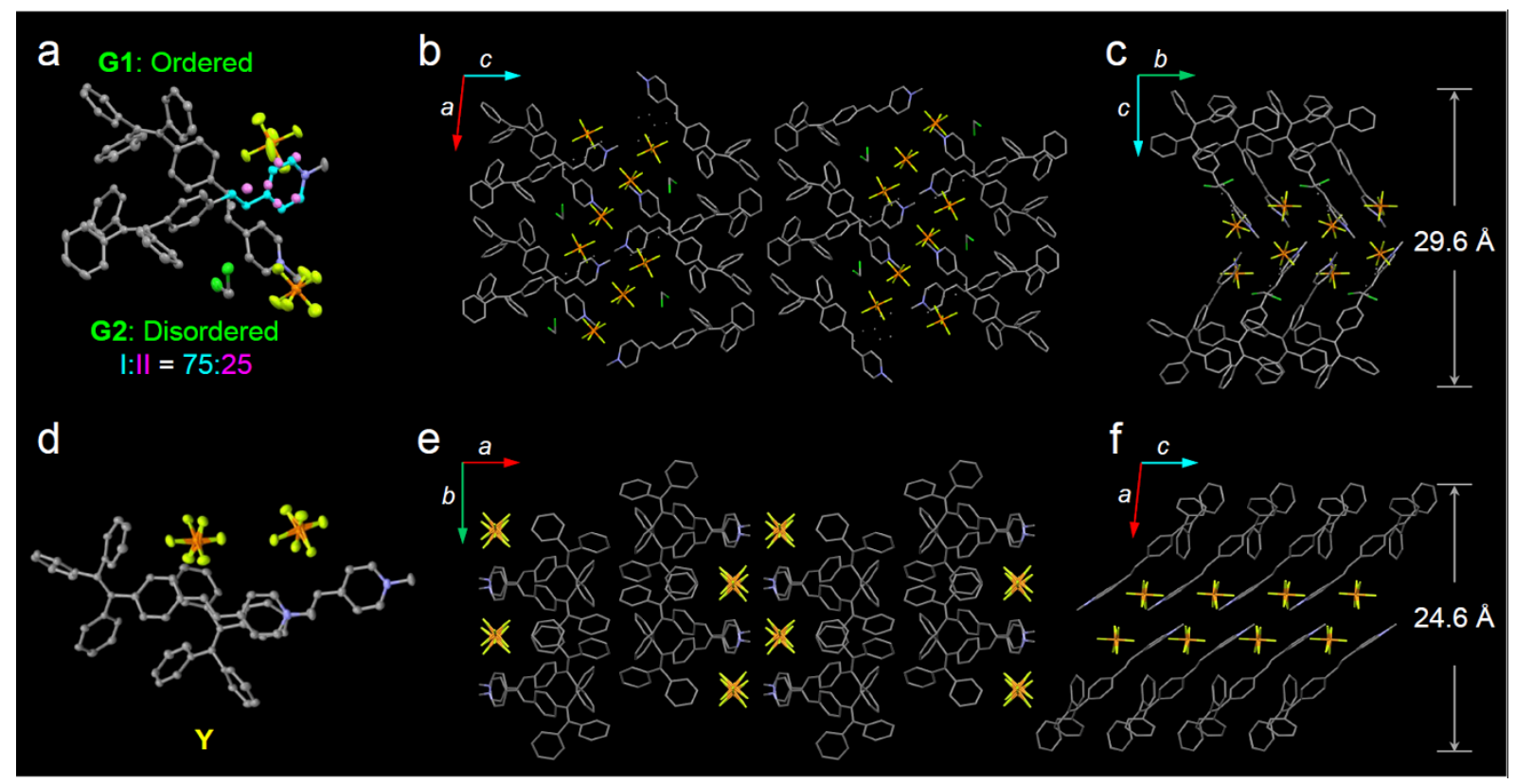

Figure 2. Crystal structures of polymorphs G and Y. (a) Ellipsoid drawing (50\% probability level) of the crystal structure of $\mathbf{G}$ adopting the cross-packing mode. A dimer unit consisting of the crystallographically ordered unit (G1) and disordered unit (G2) with two conformations. Conformers I and II are colored with blue and pink, respectively. Occupancies of the conformers I and II are $75 \%$ and $25 \%$, respectively. (b) Crystal structure of $\mathbf{G}$ depicted as stick model viewed along the $b$ axis. (c) Crystal structure of $\mathbf{G}$ viewed along the $a$ axis. (d) Ellipsoid drawing 
of the crystal structure of $\mathbf{Y}$ adopting the parallel-stacking mode. (e, f) Crystal structures of $\mathbf{Y}$. The hydrogen atoms in $\mathbf{G}$ - and $\mathbf{Y}$-crystals are omitted for clarity.

The formulae of the G-crystals and Y-crystals are $\mathrm{C}_{69} \mathrm{H}_{58} \mathrm{Cl}_{2} \mathrm{~F}_{12} \mathrm{~N}_{2} \mathrm{P}_{2}$ (TPE-EP dimer with dichloromethane at a ratio of $1: 2$ ) and $\mathrm{C}_{34} \mathrm{H}_{28} \mathrm{~F}_{6} \mathrm{NP}$, respectively (Figure 2a-f, Table S2). In the case of G-crystals, $\mathbf{G} 1$ and $\mathbf{G} 2$ form a pair in which two molecules are cross packed with two TPE units arranged on the same sides (Figure 2a). No apparent overlap of their $\pi$-planes was found (Figure S3, Supporting Information). In addition, an orientational disorder (G2) was observed in the crystal structure because of the adoption of two crystallographically independent conformations with occupancies of 75:25 (I:II). The ordered (G1) and disordered (G2) conformers are alternately placed from row to row (Figure S4). Besides, infinite channels exist in G-crystals, which are enough to accommodate small solvent molecules in the crystalline lattice (Figure 2b). ${ }^{36}$ The presence of voids in G-crystals can explain the observed larger crystal volume (6121.68 $\AA^{3} / 8$ molecules) relative to that of Y-crystals (2845.01 $\AA^{3} / 4$ molecules) (Table S2). The inclusion of dichloromethane solvent in G-crystals results in an increase of molecular flexibility and decrease of radiative transition. ${ }^{37}$ These cross-stacking dimers account for the needlelike crystals (Figure 1b), ${ }^{38}$ which are stabilized by multiple intermolecular interactions (Figure S5).

Compared with G-crystals, the increased overlapping between the pyridine rings and the double bonds of $\mathbf{Y}$-crystals accounts for the stronger intermolecular interactions to induce a redshift in emission (Figure 2d, Figure S3). The two adjacent molecules adopt comparatively parallel head-to-head arrangement, in which one molecule slides to its neighbor along the molecular long axis. They show strong 2D growth trend along the crystallographic a-b plane (Figure 2e) that results in the formation of plate-like crystals (Figure 1b). ${ }^{39}$ Meanwhile, more and stronger interatomic hydrogen bonds $(\mathrm{C}-\mathrm{H} \cdots \mathrm{F}: 2.041-2.555 \AA$, Figure S6) are observed in 
Y-crystals than that in G-crystals (C-H $\cdots \mathrm{F}: 2.413-2.666 \AA$, Figure S5), which further rigidify the molecular conformation and inhibit intramolecular rotations. These results are consistent with the higher $\Phi_{\mathrm{F}}(0.32)$ of $\mathbf{Y}$-crystals than $\mathbf{G}$-crystals $\left(\Phi_{\mathrm{F}}=0.21\right.$, Table S1). The TPE-EP molecule is characterized by a segregated structure in $\mathbf{G}$ - and $\mathbf{Y}$-crystals, which aggregates into layered structures with separated hydrophobic and hydrophilic units. The period distances of the layer structures are $24.6 \AA$ for the Y-crystals (Figure $2 \mathrm{f}$ ) and $29.6 \AA$ for the G-crystals (Figure 2c), respectively.

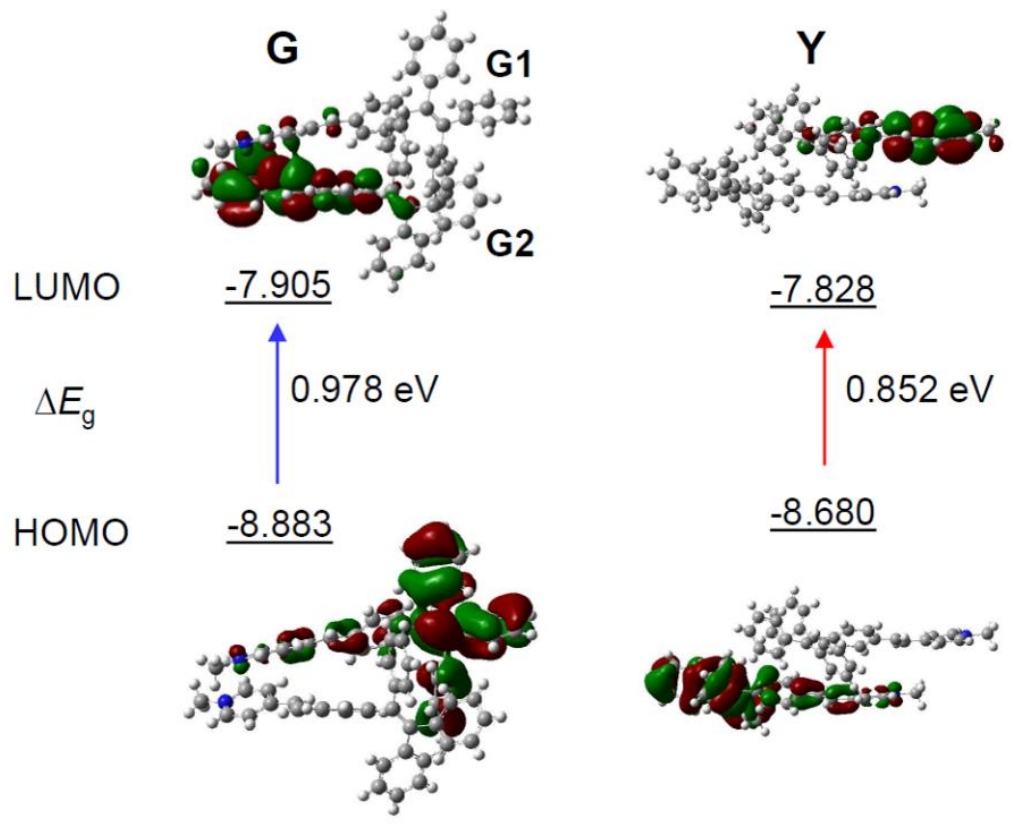

Figure 3. Molecular orbitals of TPE-EP dimer. Calculated HOMO and LUMO levels for (left) G- and (right) Y-dimer selected from single crystals. The calculation is obtained from B3LYP/6-311G(d,p) basis set by Gaussian 09 program.

To gain more insight into the mechanism of the fluorescence properties of different polymorphs of G- and Y-crystals, theoretical calculations were carried out using the density functional theory (DFT). ${ }^{40}$ The geometry of the monomer and dimer at the ground state was 
constructed based on the conformers of single crystals. As expected, the HOMO (highest occupied molecular orbital) is mainly contributed by the orbitals of the TPE unit, while the LUMO (lowest unoccupied molecular orbital) is mainly localized on the pyridinium moiety (Figure 3, Figure S7). Such a D-A structure imparts TPE-EP with solvatochromic effect (Figure S8). ${ }^{29}$ Unlike the TPE-EP monomers in crystals (Figure S7), the calculated energy gap $\left(\Delta E_{\mathrm{g}}\right)$ of Y-dimer $(0.852 \mathrm{eV})$ is smaller than that of $\mathbf{G}$-dimer $(\mathbf{G} 1 / \mathbf{G} 2,0.978 \mathrm{eV})$ (Figure 3). The change of the energy gap further suggests that intermolecular interactions exert significant effects on the emission red-shift from $\mathbf{G}$ - to $\mathbf{Y}$-crystals. ${ }^{41}$ Therefore, intervening the crystallization process at the nanometer scale provides an opportunity to control the polymorphism in the condensed state, ${ }^{42}$ notably the molecular packing and interactions between them.
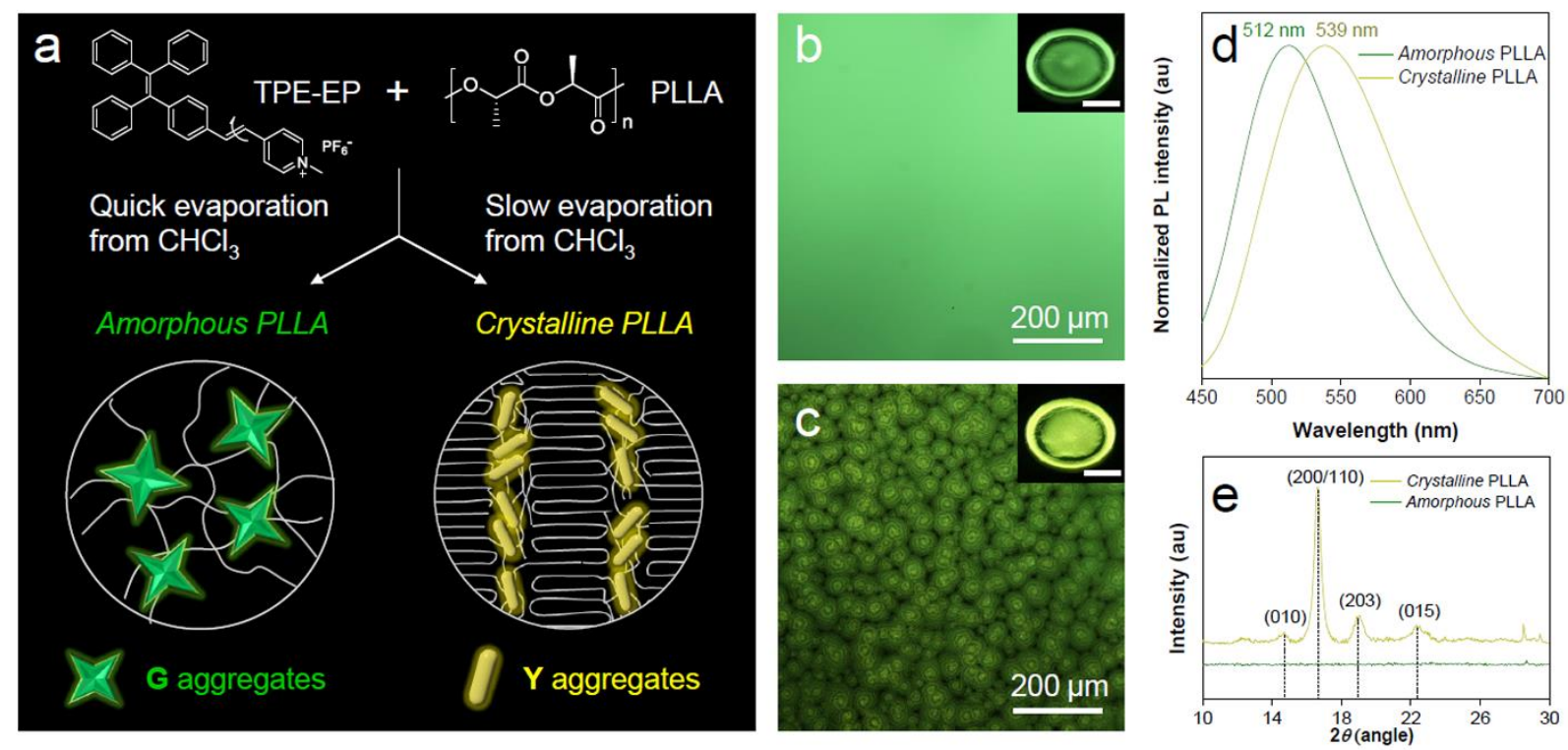

Figure 4. Polymer phase determination by TPE-EP. (a) Preparation of amorphous and crystalline PLLA embedded with TPE-EP. Schematic representation of microenvironmentsensitive fluorescence of TPE-EP in different polymer phases. (b, c) (insets) Fluorescent photos of (b) amorphous and (c) crystalline polymer films and their corresponding magnified images. Excitation wavelength: $365 \mathrm{~nm}$. Scale bar: $1 \mathrm{~cm}$. (d) Normalized PL spectra and (e) WAXD patterns of PLLA films embedded with TPE-EP at different phases. The excitation wavelength was $405 \mathrm{~nm}$. 
Polymorph selectivity in polymer phases. PLLA is a biodegradable and bio-based polymer, and is the product resulting from polymerization of L-lactide (Figure 4a). It shows potential to replace traditional petroleum-based polymeric materials. The semi-crystalline morphology of PLLA is a critical factor that influences the degradation rate, mechanical, barrier, and thermal properties. $^{43}$ The fluorescence of TPE-EP in PLLA network is shown in Figure 4a-d. The respective amorphous (Figure 4b) and crystalline (Figure 4c) PLLA films were produced by evaporative crystallization. In the amorphous region, the random molecular jumble lets the chains to cross each other, affording amorphous polymer network with flexibility and elasticity (Figure 4a, Figure S9). The incorporated TPE-EP molecules in the mobile amorphous region are allowed to orient and pack into aggregates with a structure similar to that of G-crystals (Figure $4 \mathrm{a} ; 4 \mathrm{~b}$, inset) due to the presence of large free volumes in the amorphous polymer network. ${ }^{44}$ Oppositely, in the crystalline region, the molecular chains are largely locked in place against one another and folded into crystalline lamellae, giving materials strength and rigidity (Figure 4a, Figure S9). Lamellar morphology is a periodic structure of crystalline and amorphous layers. ${ }^{45}$ Because of the nanoconfined space of the rigid amorphous phase $(\sim 5 \mathrm{~nm}$, Figure S10) between two crystalline layers, the included TPE-EP molecules in Y-aggregations are kinetically-trapped in confined space (Figure $4 \mathrm{a} ; 4 \mathrm{c}$, inset). ${ }^{42,46}$

When observed under fluorescent microscope, the homogenous amorphous film shows green emission, while the crystalline film composed of inter-linked spherulites exhibits yellow emission (Figure 4b, c). The fluorescence properties of above two films are further confirmed by photoluminescence (PL) spectroscopy (Figure 4d). It is found that PL spectra of amorphous PLLA film shows an emission maximum at $512 \mathrm{~nm}$, while the crystalline PLLA film exhibits emission maximum at $539 \mathrm{~nm}$. Both values are very close to those of $\mathbf{G}-\left(\lambda_{\max }=507 \mathrm{~nm}\right)$ and Y-crystals $\left(\lambda_{\max }=543 \mathrm{~nm}\right)$. The wide-angle XRD (WAXD) measurements of these two polymer films are performed, further confirming a significant difference in PLLA polymer chain order 
between the amorphous and crystalline states (Figure 4e). A broad and structure-less pattern was observed for amorphous PLLA, while the WAXD pattern of the crystalline PLLA shows many sharp peaks that correspond to the $\alpha$-crystalline phase. ${ }^{47}$ By varying the dye ratio in the polymer matrix from 0.1 to $0.001 \mathrm{wt} \%$, analogous experiments were conducted to prepare series of amorphous and crystalline PLLA films (Figure S11). Results showed that the emission maximum exerted little change at different TPE-EP concentrations. These data suggest that the aggregation states of guest TPE-EP molecules are mainly dependent on the microenvironment of their host polymer, showing a readily detectable color change to distinguish between amorphous and crystalline PLLA.

Crystalline phase of PLLA comprises of stacked lamellas and rigid amorphous regions sandwiched between the lamellas. Embedded TPE-EP molecules are distributed in these amorphous regions as they are driven out of lamellas during crystallization. The mobility of polymer chains in this amorphous region is hindered by the surrounding crystalline region as a polymer molecule changes conformation from crystalline to amorphous phase. ${ }^{48}$ Consequently, the rigidity of lamellar amorphous region is higher compared with the mobile amorphous region surrounding the crystalline spherulites. Furthermore, the lamellar amorphous region has a thickness of $\sim 5 \mathrm{~nm}$ (Figure S10). These factors synergistically contribute to the formation and stabilization of $\mathbf{Y}$-crystals in the crystalline phase. Nanoconfined spacing between lamellas can be a favorable factor for the formation of densely packed $\mathbf{Y}$-crystals (unit cell volume is smaller compared to G-crystals). Meanwhile, rigid environment between lamellas can further stabilize and glassify the metastable $\mathbf{Y}$-crystals.

The incompatibility between amphiphilic TPE-EP and hydrophobic PLLA polymer chains results in formation of the nanocrystalline-like aggregates rather than amorphous aggregates in the polymer matrix (Figure S12). The formation of nanocrystals can be explained by the segregated structure of TPE-EP, which forces the molecules to align in the same direction to 
take ordered organizations (Figure S13). ${ }^{33,49,50}$ In addition, the stability of TPE-EP nanoaggregates of $\mathbf{G}$ - and $\mathbf{Y}$-crystals in the polymer matrix were also investigated by monitoring the PL spectra for a time period (Figure S14). The stable PL spectra demonstrate the excellent photostability of the fluorescent films at ambient temperature.
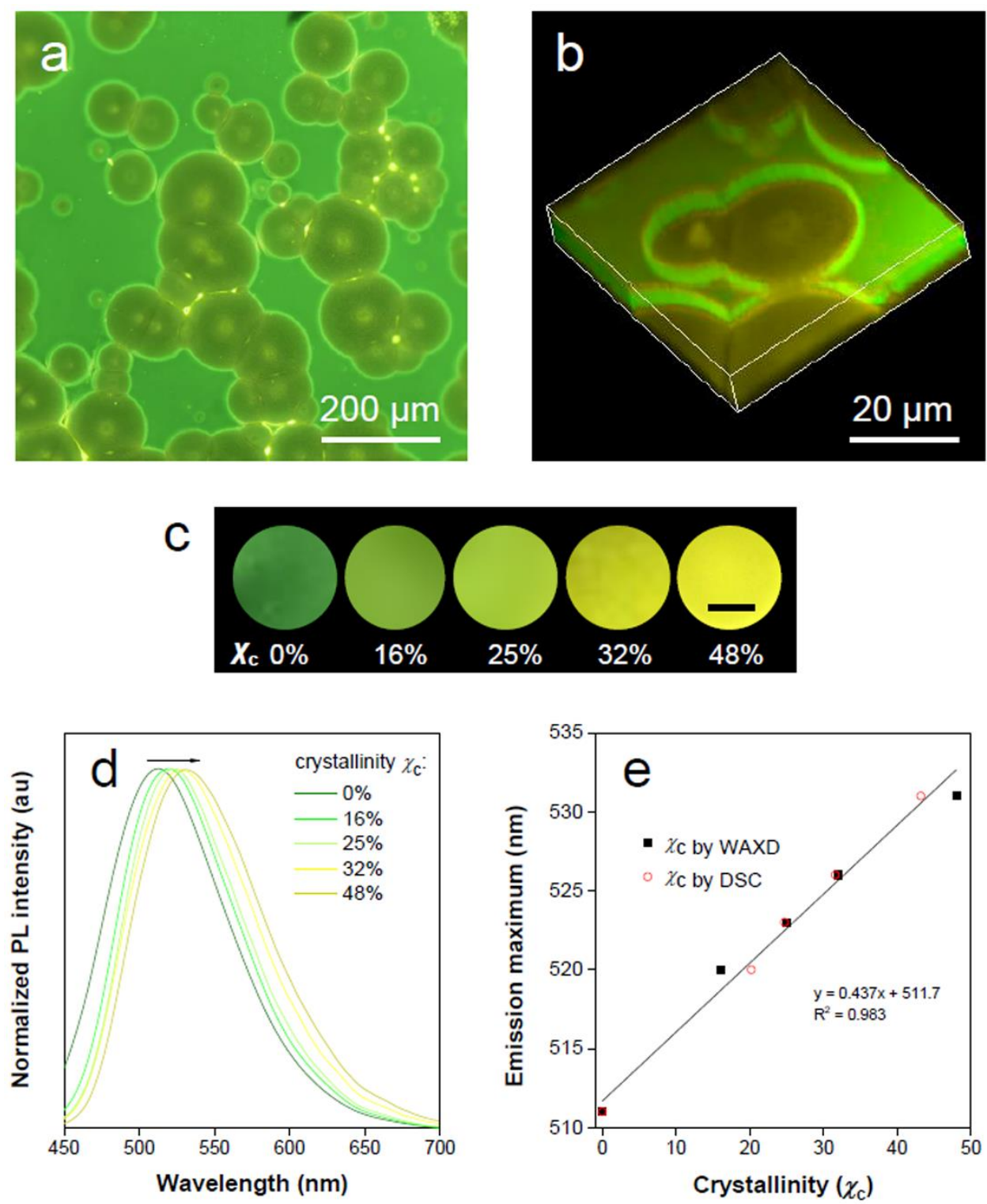

Figure 5. Polymer crystallinity visualization. (a) Overview fluorescence micrograph of the outer surface of the PLLA film with a mixture of crystalline and amorphous regions. (b) Confocal fluorescent 3D images of crystalline spherulites under $405 \mathrm{~nm}$ laser irradiation. (c) Fluorescent images of TPE-EP-labelled PLLA at various degrees of crystallinity taken under $365 \mathrm{~nm}$ UV light irradiation. The scale bar is $5 \mathrm{~mm}$. (d) Normalized PL spectra of TPE-EP- 
labelled PLLA films at various polymer crystallinity. Excitation wavelength: $405 \mathrm{~nm}$. (e) Correlation of the emission maximum with estimated crystallinity with linear-fitting curves.

Crystallinity visualization. The microphase structure of a semi-crystalline polymer is established during materials processing, which is responsible for full understanding of its physical properties. Polymer films were obtained via controlled evaporative crystallization. The 2D and 3D microscopic images of the semi-crystalline PLLA films are shown in Figure 5a and b. Through careful but relatively rapid crystallization from chloroform solution, a film with a mixture of crystalline and amorphous regions was produced (Figure 5a). Numerous spherulites with circular shapes and yellow emission are apparently recognized and are randomly distributed within the amorphous green region. The distinct fluorescent emission of the polymer crystalline/amorphous phases lights up the whole morphology of the polymer film. In addition, noticeable boundary rings in bright emission surrounding the crystalline spherulites are clearly observed, which are not easily distinguished in the bright-field image (Figure S15). The interior morphology can be directly observed through CFM based on the different emission properties of G- and Y-aggregates. The confocal image ( $X Y$ plane) was obtained using a linear unmixing method (Figure S16), ${ }^{51}$ showing excellent consistency with the observation under fluorescence microscopy. A total of 22 images in the $X Y$ plane were collected at different depths using Zscan technique, resulting in an architecture of PLLA spherulites in the thin film (Figure 5b, Figure S17). Fluorescence inspection reveals that the noticeable bright rings are interfaces between the amorphous and crystalline polymer regions. Scanning electron microscopy (SEM) was further used to verify the interior morphology (Figure S18). With assistance of solvent (acetone) processing, the amorphous region was etched out to create a pseudo-3D construction. ${ }^{52}$ The SEM image (Figure S18) of the etched film surface shows apparent circular 
spherulites and crystalline-amorphous interface, which are coincided with those in fluorescent images.

The ratio of G-to-Y aggregates of TPE-EP in respective amorphous and crystalline phases could be modified by the degree of polymer crystallinity at different processing conditions, to tune the luminescence response of the polymers. Figure $5 \mathrm{c}$ shows the fluorescence response of TPE-EP-labelled PLLA films with crystallinity ranging from $0 \%$ to $48 \%$. The crystallinity $\left(\chi_{\mathrm{c}}\right)$ was estimated by WAXD and differential scanning calorimetry (DSC), respectively (Figure S19 and Table S3). A progressive red-shift from green to yellow was observed, providing a visible crystallinity-detection platform. The fluorescence properties of the above films are further studied by PL spectroscopy. Figure 5d exhibits the normalized PL spectra of TPE-EP-embedded PLLA films at crystallinity of $0 \%, 16 \%, 25 \%, 32 \%$ and $48 \%$, respectively. With an increase in $x_{\mathrm{c}}(0-48 \%)$, the PL spectrum is progressively red-shifted from $511 \mathrm{~nm}$ to $531 \mathrm{~nm}$. A linear relationship exists between the emission maximum and $\chi_{\mathrm{c}}$, indicating that TPE-EP can serve as colorimetric marker for crystallinity visualization (Figure 5e). The crystallinity calculated from DSC and PL data also conform well to a linear relationship. Such a calibration line enables quantitative measurements of $x_{\mathrm{c}}$ from the PL change. The results indicate that our AIE marker could not only tell the microscale polymer crystalline phase distribution within the sample, but also provide the average crystallinity over the bulk material. In addition, benefit from the sensitivity of fluorescence light, our quantitative crystallinity measurements are applicable to the sample with a size even down to micrometer. 

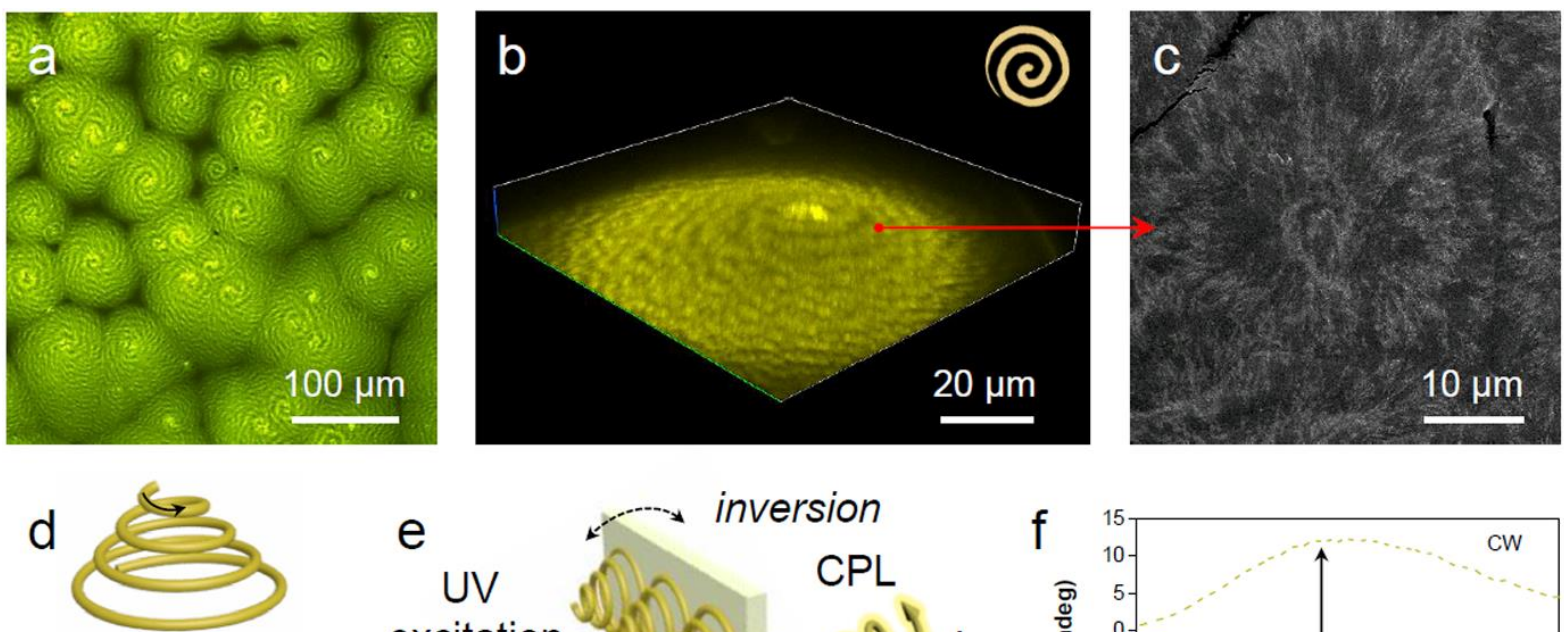

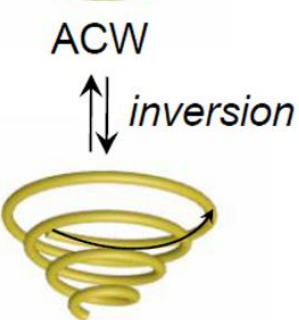

$\mathrm{CW}$

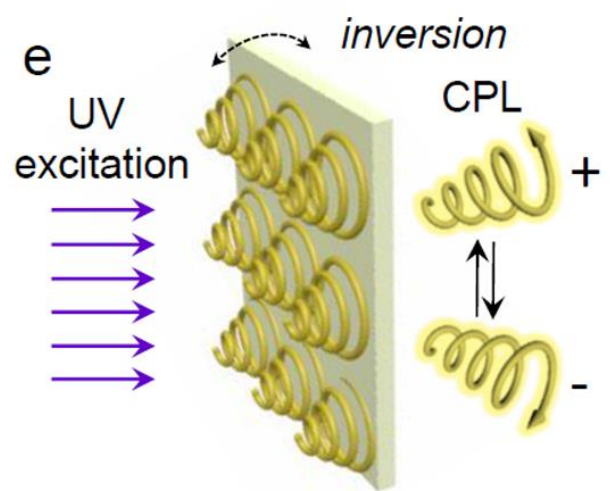

polymer spherulites

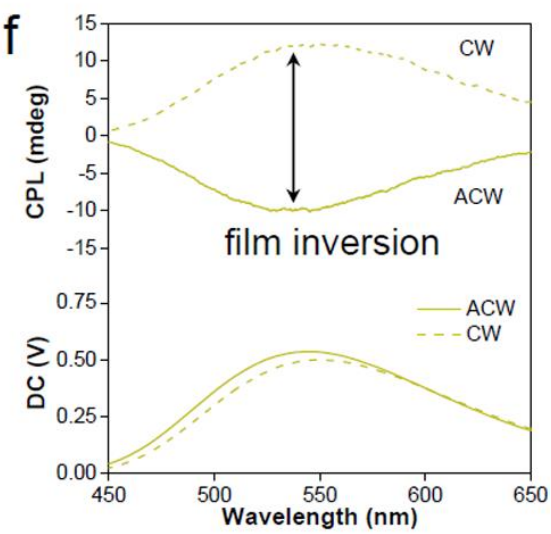

Figure 6. Spatial mapping at microscopic scale. (a) Representative microscopic fluorescent (2D) and (b) confocal (3D) representation of the PLLA spherulites with alternative bright and dark yellow spirals in anticlockwise direction. (c) SEM micrograph of a similar spherulite illustrating the interior morphological structure after acetone etching. The red arrow indicates the correlated locations between fluorescent confocal image and electron micrograph. (d) Enantiomeric chirality switching of the spiral spherulites by film inversion. (e) The spiral spherulites are organized to form a crystalline polymer film, which act as a chiral medium to generate CPL by preferential reflection of co-handed CPL. (f) Enantiomeric CPL switch upon film inversion.

Spatial visualization. In the process of evaporative crystallization, because of the imbalanced stress at opposite folding surfaces of PLLA, twisting of the crystalline lamellas occurs (Figure S20). ${ }^{53}$ As a result, banded spherulites with a specific handedness are developed because of the radial growth of the helical lamellae from edge-on gradually twisting to flat-on orientations. ${ }^{54}$ 
Slow evaporation process was then conducted to produce banded PLLA spherulites with long pitch. ${ }^{55}$ The fluorescent microscopic image in Figure 6a shows the formation of spherulites with alternating bright- and dark-yellow spirals in anti-clockwise (ACW) direction. Such microstructure of PLLA spherulites was further verified by confocal analysis (Figure 6b; Figure S21). A 3D spherulites with Archimedean spiral organization is presented in the upward vertical direction, which is attributed to the non-diametric sections of 3D spherulites. ${ }^{55}$ The SEM image of the acetone-etched interiors of PLLA spherulite and demonstrates interesting correlations between emission and lamellae assembly (Figure 6c). ${ }^{56}$ The ridge band in bright-yellow is composed of lamellar bundles, and nano-cracks between the parallel edge-on crystals are visible on the ridge band in SEM images (after solvent etching, Figure S22). Meanwhile, the smooth valley band in dark-yellow is mostly filled by flat-on lamellas. ${ }^{57}$ The bright-yellow emission of the ridge band is mostly ascribed to optical scattering when light penetrates through these fractured and rough places. The ridges and valleys in SEM images conform well to the brightand dark-yellow spirals observed in fluorescent and confocal microscope. Fluorescence imaging provides a straightforward and non-invasive method to visualize both surface and interior of the polymer materials.

The 3D spirals highlighted with yellow emission in polymer spherulites present a good chiroptical activity ${ }^{58}$ which can divide incident light into left/right-handed circularly polarized light (CPL) components by selective reflection and transmission. ${ }^{59} \mathrm{~A}$ reversal of the direction provides a clockwise $(\mathrm{CW})$ spiral, resulting in a mirror image of the ACW spiral (Figure 6d). Upon UV excitation, the AIE-labelled polymer films with chiral structured spherulites are supposed to have an intrinsic ability for CPL generation (Figure 6e). The film reversal would result in chirality switching while keeping the enantiomeric symmetry. In order to demonstrate the relationship between microscopic morphologies described above and macroscopic optical properties, CPL spectroscopy was conducted to study the chiroptical activity of such polymeric 
films. The magnitude of CPL can be evaluated by the luminescence dissymmetry factor (glum), which equals to $2\left(I_{\mathrm{L}}-I_{\mathrm{R}}\right) /\left(I_{\mathrm{L}}+I_{\mathrm{R}}\right)$, where $I_{\mathrm{L}}$ and $I_{\mathrm{R}}$ refer to the intensity of left- and right-handed CPL, respectively. ${ }^{60,61}$ In Figure 6f, a negative CPL response with an emission maximum at $\sim 540 \mathrm{~nm}$ with a glum of $\sim-1.6 \times 10^{-3}$ was observed. Upon flipping the sample, a positive CPL response with glum of $\sim 1.6 \times 10^{-3}$ was detected (Figure S23). The induced CPL spectra show mirror image upon film inversion, which is modulated by the spiral morphology of the spherulites (Figure S23). In this regard, the fluorescent AIE marker in polymer matrix simultaneously offers understanding of the spatial organization of the crystalline polymer lamellas as well as provides a correlation between spiral morphological information and polarized optical signal.

\section{CONCLUSIONS}

In summary, we have demonstrated how polymorphic TPE-EP can be a unique "built-in" marker to visualize polymer morphologies extending over several length scales. The information on polymer phases (amorphous and crystalline) is marked with distinct color based on the polymorphic states of TPE-EP in specific polymer phases. As a result, the complex hierarchical organization of polymer morphology is transformed into optical signals, which can be told by color and polarization. We envision that the present concept would be applicable to diverse commodity polymers through proper molecular structure design. These morphological sensing properties have potential for in-situ monitoring polymer manufacturing process, in turn, to predict materials physical properties. Furthermore, the synergic effects between luminescent molecules and micro-structured polymers account for versatile luminescence manipulation, including intensity, color, and polarization, providing continuous emissive materials in developing foldable devices and wearable systems. 


\section{Methods}

Materials. TPE-EP was synthesized according to the synthetic methods reported previously. ${ }^{62}$ All starting materials and reagents were purchased from commercial suppliers and used as received. The PLLA $\left(M_{\mathrm{w}}=8.9 \times 10^{4} \mathrm{~g} \mathrm{~mol}^{-1}\right.$, PDI $\left.=1.19\right)$ was purified by dissolving in chloroform and precipitated in methanol and was dried in a vacuum oven before use.

Single crystal and X-ray analysis. All crystals in this work were obtained at room temperature. G-crystals were cultivated through vapor diffusion of dichloromethane/hexane mixtures of TPE. Y-crystals were prepared through slow evaporation of tetrahydrofuran (THF)/hexane mixtures. O-crystals were obtained through slow evaporation of THF mixtures. Measurement of the single crystals were collected on a SuperNova Dual Atlas diffractometer using graphite monochromated $\mathrm{Cu} \mathrm{K} \alpha$ radiation $(\lambda=1.54184 \AA$ ). The diffraction data were collected at $100 \mathrm{~K}$ and the crystallographic parameters are summarized in Table S2. The structure was solved with the ShelXS structure solution program using direct methods in Olex $2^{63}$ and refined with ShelXL refinement package using Least Squares minimization. Non-hydrogen atoms were refined anisotropically while hydrogen atoms were refined using the riding model.

Preparation of TPE-EP labelled PLLA film. Typical preparation procedures are as follows. TPE-EP was dissolved in THF to generate a $1 \mathrm{mg} \mathrm{mL}^{-1}$ stock solution. $50 \mu \mathrm{L}$ of the above TPEEP solution was mixed with $50 \mathrm{mg}$ of PLLA in chloroform $\left(10 \mathrm{mg} \mathrm{mL}^{-1}\right)$ under stirring. Unless specified otherwise, the content of TPE-EP in PLLA matrix was controlled to be $0.1 \mathrm{wt} \%$. Afterward, the mixed solution was put in a covered weighing bottle with a radius and height of $2.5 \mathrm{~cm}$ and $3.0 \mathrm{~cm}$, respectively. Crystallization of PLLA was occurred by controlling the solvent evaporation rate at ambient temperature. Then, the thin PLLA film was peeled off from the glass bottle for further study. 
CFM imaging. CFM measurements were carried out on a Zeiss LSM7 DUO confocal microscope. For TPE-EP-labeled PLLA film with a mixture of amorphous and crystalline phases, CFM images were first obtained through two-channel scanning under laser excitation of $405 \mathrm{~nm}$. Channel one was selected between 410-516 nm and channel two was selected between 518-688 $\mathrm{nm}$. The obtained images were linearly unmixed ${ }^{64}$ using ZEN software into two components, each of which was respectively assigned by green and yellow as shown in Figure S16 and S17. For TPE-EP-labelled crystalline PLLA film, CFM images were obtained under laser excitation of $405 \mathrm{~nm}$, and the optical signal was selected between 400-700 nm (Figure S21).

Measurements. Molecular weight and polydispersity index (PDI) of purified PLLA was determined by Waters Association Gel Permeation Chromatography (GPC) system equipped with UV detectors. Differential Scanning Calorimetry (DSC) measurements were conducted using a TA DSC Q1000 under nitrogen flow at a heating rate of $10^{\circ} \mathrm{C} \mathrm{min}^{-1}$. Photoluminescence (PL) spectra were measured on a Horiba Fluorolog-3 spectrofluorometer. Quantum yields of the solid samples and polymer films were determined on a Horiba Quanta using a calibrated integrating sphere. Fluorescence lifetime measurements were conducted on a FLS 980 spectrometer. Fluorescent and optical microscopy images were captured on a Nikon Eclipse 80i microscope. WAXD analysis was conducted on a X'pert PRO, PANanalytical diffractometer using $\mathrm{Cu}-\mathrm{K}$ radiation. Small-angle $\mathrm{X}$-ray scattering (SAXS) experiments were performed on a SAXSess instrument (Anton Paar). Nanoindentation tests were conducted using a Hysitron TI950 TriboIndenter. SEM images were obtained on a JEOL-7100F SEM. Normal photographs were taken on Canon 7D camera under $365 \mathrm{~nm}$ UV illumination. CPL spectra were carried out on JASCO CPL-300, the measurement conditions of CPL are listed in Table S4. 
Crystallinity measurements. Percentage crystallinities $\left(\chi_{c}\right)$ of the polymer films were estimated by DSC and XRD methods. For DSC measurements, crystallinities of the polymer films were calculated from DSC thermal profiles according to equation (1).

$$
\chi_{\mathrm{c}}(\%)=\frac{\Delta \mathrm{H}_{m}-\Delta \mathrm{H}_{c}}{\Delta \mathrm{H}_{m}^{0}} \times 100 \%
$$

$\Delta \mathrm{H}_{\mathrm{m}}$ is melting enthalpy and $\Delta \mathrm{H}_{\mathrm{c}}$ is exothermal enthalpy arising from crystallization in the polymer film during heating process. $\Delta \mathrm{H}^{0}{ }_{\mathrm{m}}$ is the melting enthalpy of PLLA crystal, $106 \mathrm{~J} \mathrm{~g}^{-1} \cdot{ }^{65}$ DSC thermographs of tested films are shown in Figure S19 and thermal profiles indicating enthalpies and temperatures of melting and crystallization of the films are shown in Table S3. For XRD measurements, crystallinities of the polymer films were calculated by dividing the area under the crystalline peaks to overall area of the diffractometer after subtraction of the background signal. Strong peak at $2 \theta$ of $16.7^{\circ}$ was originated from $(200) /(110)$ planes, and relatively smaller peak at $2 \theta$ of $19.0^{\circ}$ from (203) planes was selected as crystalline peaks for fitting.

Lamellae thickness calculation. Lamellae thickness and long period were calculated from SAXS patterns using one-dimensional (1D) correlation function. ${ }^{66}$ The normalized 1D correlation function was extracted from Lorentz-corrected experimental SAXS results by a Fourier transform by equation (2).

$$
\gamma_{x}=\frac{\int_{0}^{\infty} I(q) q^{2} \cos (q x) d q}{\int_{0}^{\infty} I(q) q^{2} d q}
$$

The long period $L$ is the first maximum value of first oscillation, and the thickness of the thinner phase ( $L_{\mathrm{t}}$, determined as amorphous phase) is the intercept between level of the first minimum and the extrapolation of linear part of the correlation function as labelled in Figure S10. 
Single crystal data. CCDC \#1917740 for G-crystals and 1917741 for Y-crystals contains the supplementary crystallographic data for this paper. These data can be obtained free of charge from The Cambridge Crystallographic Data Centre via www.ccdc.cam.ac.uk/data_request/cif.

\section{ASSOCIATED CONTENT}

\section{Supporting Information.}

The Supporting Information is available free of charge on ACS Publications website at DOI: XXX. Supporting Information contains properties of single crystals of TPE-EP, characterizations of polymer morphologies (PDF).

Tables S1-S4 (PDF)

Figures S1-S23 (PDF)

\section{AUTHOR INFORMATION}

Corresponding Author:

*E-mail: cyh@dhu.edu.cn (Y. H. Cheng)

*E-mail: tanbenz@ust.hk (B. Z. Tang)

\section{Author Contributions}

The manuscript was written through contributions of all authors. All authors have given approval to the final version of the manuscript. ${ }^{\perp}$ These authors contributed equally.

\section{Notes}

The authors declare no competing financial interest. 


\section{ACKNOWLEDGMENT}

This work was partially supported by the National Science Foundation of China (51603035, 21788102, 21490570 and 21490574), the Research Grants Council of Hong Kong (16308116 and C6009-17G), the Innovation and Technology Commission (ITC-CNERC14SC01 and ITS/254/17), the Science and Technology Plan of Shenzhen (JCYJ20170818113602462, JCYJ20160229205601482 and JCYJ20160509170535223), Science and Technology Commission of Shanghai Municipality (16JC1400700 and 17ZR1446300), the China Postdoctoral Science Foundation (2016M591572 and 2017T100256), the Fundamental Research Funds for the Central Universities (2232018D3-01), and State Key Laboratory for Modification of Chemical Fibers and Polymer Materials, Donghua University.

\section{REFERENCES}

1. Autumn, K.; Liang, Y. A.; Hsieh, S. T.; Zesch, W.; Chan, W. P.; Kenny, T. W.; Fearing, R.; Full, R. J. Adhesive force of a single gecko foot-hair. Nature 2000, 405, 681-685.

2. Kamat, S.; Su, X.; Ballarini, R.; Heuer, A. H. Structural basis for the fracture toughness of the shell of the conch Strombus gigas. Nature 2000, 405, 1036-1040.

3. Jiang, L.; Zhao, Y.; Zhai, J. A Lotus-Leaf-like Superhydrophobic Surface: A Porous Microsphere/Nanofiber Composite Film Prepared by Electrohydrodynamics. Angew. Chem. Int. Ed. 2004, 43, 4338-4341.

4. Wegst, U. G. K.; Bai, H.; Saiz, E.; Tomsia, A. P.; Ritchie, R. O. Bioinspired structural materials. Nat. Mater. 2014, 14, 23-36.

5. Meyers, M. A.; McKittrick, J.; Chen, P.-Y. Structural Biological Materials: Critical Mechanics-Materials Connections. Science 2013, 339, 773-779.

6. Campoy-Quiles, M.; Ferenczi, T.; Agostinelli, T.; Etchegoin, P. G.; Kim, Y.; Anthopoulos, T. D.; Stavrinou, P. N.; Bradley, D. D. C.; Nelson, J. Morphology evolution via self-organization and lateral and vertical diffusion in polymer:fullerene solar cell blends. Nat. Mater. 2008, 7, 158-164.

7. Geyer, R.; Jambeck, J. R.; Law, K. L. Production, use, and fate of all plastics ever made. Sci. Adv. 2017, 3, e1700782. 
8. Cui, K.; Ma, Z.; Tian, N.; Su, F.; Liu, D.; Li, L. Multiscale and Multistep Ordering of Flow-Induced Nucleation of Polymers. Chem. Rev. 2018, 118, 1840-1886.

9. Xu, Y.; Kraemer, D.; Song, B.; Jiang, Z.; Zhou, J.; Loomis, J.; Wang, J.; Li, M.; Ghasemi, H.; Huang, X.; et al. Nanostructured polymer films with metal-like thermal conductivity. Nat. Commun. 2019, 10, 1771.

10. Liu, Y.; Zhao, J.; Li, Z.; Mu, C.; Ma, W.; Hu, H.; Jiang, K.; Lin, H.; Ade, H.; Yan, H. Aggregation and morphology control enables multiple cases of high-efficiency polymer solar cells. Nat. Commun. 2014, 5, 5293.

11. Athawale, A. A.; Kulkarni, M. V. Polyaniline and its substituted derivatives as sensor for aliphatic alcohols. Sens. Actuators B. Chem. 2000, 67, 173-177.

12. Kimata, S.; Sakurai, T.; Nozue, Y.; Kasahara, T.; Yamaguchi, N.; Karino, T.; Shibayama, M.; Kornfield, J. A. Molecular Basis of the Shish-Kebab Morphology in Polymer Crystallization. Science 2007, 316, 1014-1017.

13. Panova, O.; Chen, X. C.; Bustillo, K. C.; Ophus, C.; Bhatt, M. P.; Balsara, N.; Minor, A. M. Orientation mapping of semicrystalline polymers using scanning electron nanobeam diffraction. Micron 2016, 88, 30-36.

14. Qi, J.; Sun, C.; Zebibula, A.; Zhang, H.; Kwok, R. T. K.; Zhao, X.; Xi, W.; Lam, J. W. Y.; Qian, J.; Tang, B. Z. Real-Time and High-Resolution Bioimaging with Bright AggregationInduced Emission Dots in Short-Wave Infrared Region. Adv. Mater. 2018, 30, 1706856.

15. Dean, K. M.; Palmer, A. E. Advances in fluorescence labeling strategies for dynamic cellular imaging. Nat. Chem. Biol. 2014, 10, 512-523.

16. Rob, A.; Frederik, V.; Johan, H.; Frans C., D. S.; Dirk E., D. V.; Maarten. B. J. R. ThreeDimensional Visualization of Defects Formed during the Synthesis of Metal-Organic Frameworks: A Fluorescence Microscopy Study. Angew. Chem. Int. Ed. 2013, 125, 419-423.

17. Dekkers, J. F.; Alieva, M.; Wellens, L. M.; Ariese, H. C. R.; Jamieson, P. R.; Vonk, A. M.; Amatngalim, G. D.; Hu, H.; Oost, K. C.; Snippert, H. J. G.; et al. High-resolution 3D imaging of fixed and cleared organoids. Nat. Protoc. 2019, 14, 1756-1771.

18. Kanchanawong, P.; Shtengel, G.; Pasapera, A. M.; Ramko, E. B.; Davidson, M. W.; Hess, H. F.; Waterman, C. M. Nanoscale architecture of integrin-based cell adhesions. Nature 2010, $468,580-584$.

19. Mei, J.; Leung, N. L. C.; Kwok, R. T. K.; Lam, J. W. Y.; Tang, B. Z. AggregationInduced Emission: Together We Shine, United We Soar! Chem. Rev. 2015, 115, 11718-11940. 20. He, Z.; Ke, C.; Tang, B. Z. Journey of Aggregation-Induced Emission Research. ACS Omega 2018, 3, 3267-3277. 
21. Chen, Y.; Lam, J. W. Y.; Kwok, R. T. K.; Liu, B.; Tang, B. Z. Aggregation-induced emission: fundamental understanding and future developments. Mater. Horiz. 2019, 6, 428433.

22. Dong, J.; Li, X.; Zhang, K.; Di Yuan, Y.; Wang, Y.; Zhai, L.; Liu, G.; Yuan, D.; Jiang, J.; Zhao, D. Confinement of Aggregation-Induced Emission Molecular Rotors in Ultrathin Two-Dimensional Porous Organic Nanosheets for Enhanced Molecular Recognition. J. Am. Chem. Soc. 2018, 140, 4035-4046.

23. Han, T.; Gui, C.; Lam, J. W. Y.; Jiang, M.; Xie, N.; Kwok, R. T. K.; Tang, B. Z. HighContrast Visualization and Differentiation of Microphase Separation in Polymer Blends by Fluorescent AIE Probes. Macromolecules 2017, 50, 5807-5815.

24. Iasilli, G.; Battisti, A.; Tantussi, F.; Fuso, F.; Allegrini, M.; Ruggeri, G.; Pucci, A. Aggregation-Induced Emission of Tetraphenylethylene in Styrene-Based Polymers. Macromol. Chem. Phys. 2014, 215, 499-506.

25. Guan, W.; Wang, S.; Lu, C.; Tang, B. Z. Fluorescence microscopy as an alternative to electron microscopy for microscale dispersion evaluation of organic-inorganic composites. Nat. Commun. 2016, 7, 11811.

26. Liu, S.; Cheng, Y.; Zhang, H.; Qiu, Z.; Kwok, R. T. K.; Lam, J. W. Y.; Tang, B. Z. In Situ Monitoring of RAFT Polymerization by Tetraphenylethylene-Containing Agents with Aggregation-Induced Emission Characteristics. Angew. Chem. Int. Ed. 2018, 57, 6274-6278.

27. Peng, H.-Q.; Liu, B.; Wei, P.; Zhang, P.; Zhang, H.; Zhang, J.; Li, K.; Li, Y.; Cheng, Y.; Lam, J. W. Y.; et al. Visualizing the Initial Step of Self-Assembly and the Phase Transition by Stereogenic Amphiphiles with Aggregation-Induced Emission. ACS Nano 2018, 13, 839846.

28. Wang, Z.; Nie, J.; Qin, W.; Hu, Q.; Tang, B. Z. Gelation process visualized by aggregation-induced emission fluorogens. Nat. Commun. 2016, 7, 12033.

29. Cheng, Y.; Wang, J.; Qiu, Z.; Zheng, X.; Leung, N. L. C.; Lam, J. W. Y.; Tang, B. Z. Multiscale Humidity Visualization by Environmentally Sensitive Fluorescent Molecular Rotors. Adv. Mater. 2017, 29, 1703900.

30. Ma, Z.; Wang, Z.; Teng, M.; Xu, Z.; Jia, X. Mechanically Induced Multicolor Change of Luminescent Materials. ChemPhysChem 2015, 16, 1811-1828.

31. He, Z.; Zhang, L.; Mei, J.; Zhang, T.; Lam, J. W. Y.; Shuai, Z.; Dong, Y. Q.; Tang, B. Z. Polymorphism-Dependent and Switchable Emission of Butterfly-Like Bis(diarylmethylene)dihydroanthracenes. Chem. Mater. 2015, 27, 6601-6607. 
32. Sagara, Y.; Yamane, S.; Mitani, M.; Weder, C.; Kato, T. Mechanoresponsive Luminescent Molecular Assemblies: An Emerging Class of Materials. Adv. Mater. 2016, 28, 1073-1095.

33. Yagai, S.; Okamura, S.; Nakano, Y.; Yamauchi, M.; Kishikawa, K.; Karatsu, T.; Kitamura, A.; Ueno, A.; Kuzuhara, D.; Yamada, H.; et al. Design amphiphilic dipolar $\pi$-systems for stimuli-responsive luminescent materials using metastable states. Nat. Commun. 2014, 5, 4013.

34. Cheng, Y.; Liu, S.; Song, F.; Khorloo, M.; Zhang, H.; Kwok, R. T. K.; Lam, J. W. Y.; He, Z.; Tang, B. Z. Facile emission color tuning and circularly polarized light generation of single luminogen in engineering robust forms. Mater. Horiz. 2019, 6, 405-411.

35. Ito, H.; Muromoto, M.; Kurenuma, S.; Ishizaka, S.; Kitamura, N.; Sato, H.; Seki, T. Mechanical stimulation and solid seeding trigger single-crystal-to-single-crystal molecular domino transformations. Nat. Commun. 2013, 4, 2009.

36. Fabbiani, F. P. A.; Byrne, L. T.; McKinnon, J. J.; Spackman, M. A. Solvent inclusion in the structural voids of form II carbamazepine: single-crystal X-ray diffraction, NMR spectroscopy and Hirshfeld surface analysis. CrystEngComm 2007, 9, 728-731.

37. Huang, B.; Chen, W.-C.; Li, Z.; Zhang, J.; Zhao, W.; Feng, Y.; Tang, B. Z.; Lee, C.-S. Manipulation of Molecular Aggregation States to Realize Polymorphism, AIE, MCL, and TADF in a Single Molecule. Angew. Chem. Int. Ed. 2018, 57, 12473-12477.

38. Xie, Z.; Yang, B.; Li, F.; Cheng, G.; Liu, L.; Yang, G.; Xu, H.; Ye, L.; Hanif, M.; Liu, S.; et al. Cross Dipole Stacking in the Crystal of Distyrylbenzene Derivative: The Approach toward High Solid-State Luminescence Efficiency. J. Am. Chem. Soc. 2005, 127, 14152-14153. 39. Yuan, M.-S.; Wang, D.-E.; Xue, P.; Wang, W.; Wang, J.-C.; Tu, Q.; Liu, Z.; Liu, Y.; Zhang, Y.; Wang, J. Fluorenone Organic Crystals: Two-Color Luminescence Switching and Reversible Phase Transformations between $\pi-\pi$ Stacking-Directed Packing and Hydrogen Bond-Directed Packing. Chem. Mater. 2014, 26, 2467-2477.

40. Frisch, M. J.; Trucks, G. W.; Schlegel, H. B.; Scuseria, G. E.; Robb, M. A.; Cheeseman, J. R.; Scalmani, G.; Barone,V.; Mennucci, B.; Petersson, G. A.; et al. Gaussian 09. Gaussian, Inc. Wallingford CT, 2009.

41. Dong, Y.; Xu, B.; Zhang, J.; Tan, X.; Wang, L.; Chen, J.; Lv, H.; Wen, S.; Li, B.; Ye, L.; et al. Piezochromic Luminescence Based on the Molecular Aggregation of 9,10-Bis((E)-2(pyrid-2-yl)vinyl)anthracene. Angew. Chem. Int. Ed. 2012, 51, 10782-10785. 
42. Hamilton, B. D.; Ha, J.-M.; Hillmyer, M. A.; Ward, M. D. Manipulating Crystal Growth and Polymorphism by Confinement in Nanoscale Crystallization Chambers. Acc. Chem. Res. 2012, 45, 414-423.

43. Domenek, S.; Fernandes-Nassar, S.; Ducruet, V., Rheology, Mechanical Properties, and Barrier Properties of Poly(lactic acid). In Synthesis, Structure and Properties of Poly(lactic acid), Di Lorenzo, M. L.; Androsch, R., Eds.; Springer International Publishing: Cham, 2018; pp 303-341.

44. Pan, P.; Zhu, B.; Inoue, Y., Enthalpy Relaxation and Embrittlement of Poly(1-lactide) during Physical Aging. Macromolecules 2007, 40, 9664-9671.

45. Tencé-Girault, S.; Woehling, V.; Oikonomou, E. K.; Karpati, S.; Norvez, S. About the Art and Science of Visualizing Polymer Morphology using Transmission Electron Microscopy. Macromol. Chem. Phys. 2018, 219, 1700483.

46. Ha, J.-M.; Wolf, J. H.; Hillmyer, M. A.; Ward, M. D. Polymorph Selectivity under Nanoscopic Confinement. J. Am. Chem. Soc. 2004, 126, 3382-3383.

47. Xu, H.; Zhong, G.-J.; Fu, Q.; Lei, J.; Jiang, W.; Hsiao, B. S.; Li, Z.-M. Formation of Shish-Kebabs in Injection-Molded Poly(l-lactic acid) by Application of an Intense Flow Field. ACS Appl. Mater. Inter. 2012, 4, 6774-6784.

48. Righetti, M. C., Amorphous Fractions of Poly(lactic acid). In Synthesis, Structure and Properties of Poly(lactic acid), Di Lorenzo, M. L.; Androsch, R., Eds.; Springer International Publishing: Cham, 2018; pp 195-234.

49. Li, W.-S.; Yamamoto, Y.; Fukushima, T.; Saeki, A.; Seki, S.; Tagawa, S.; Masunaga, H.; Sasaki, S.; Takata, M.; Aida, T. Amphiphilic Molecular Design as a Rational Strategy for Tailoring Bicontinuous Electron Donor and Acceptor Arrays: Photoconductive Liquid Crystalline Oligothiophene-C60 Dyads. J. Am. Chem. Soc. 2008, 130, 8886-8887.

50. Cheng, Y.-H.; Chen, W.-P.; Zheng, C.; Qu, W.; Wu, H.; Shen, Z.; Liang, D.; Fan, X.H.; Zhu, M.-F.; Zhou, Q.-F. Synthesis and Phase Structures of Mesogen-Jacketed Liquid Crystalline Polyelectrolytes and Their Ionic Complexes. Macromolecules 2011, 44, 3973-3980.

51. Zimmermann, T.; Marrison, J.; Hogg, K.; O’Toole, P. Clearing Up the Signal: Spectral Imaging and Linear Unmixing in Fluorescence Microscopy. In Confocal Microscopy: Methods and Protocols, Paddock, S. W., Eds.; Springer New York: New York, 2014; pp 129-148.

52. Madhavan Nampoothiri, K.; Nair, N. R.; John, R. P. An overview of the recent developments in polylactide (PLA) research. Bioresour. Technol. 2010, 101, 8493-8501.

53. Lotz, B.; Cheng, S. Z. D. A critical assessment of unbalanced surface stresses as the mechanical origin of twisting and scrolling of polymer crystals. Polymer 2005, 46, 577-610. 
54. Lotz, B., Crystal Polymorphism and Morphology of Polylactides. In Synthesis, Structure and Properties of Poly(lactic acid), Di Lorenzo, M. L.; Androsch, R., Eds.; Springer International Publishing: Cham, 2018; pp 273-302.

55. Li, Y.; Wu, L.; He, C.; Wang, Z.; He, T. Strong enhancement of the twisting frequency of achiral orthorhombic lamellae in poly( $\varepsilon$-caprolactone) banded spherulites via evaporative crystallization. CrystEngComm 2017, 19, 1210-1219.

56. Woo, E. M.; Lugito, G. Origins of periodic bands in polymer spherulites. Eur. Polym. J. 2015, 71, 27-60.

57. Nurkhamidah, S.; Woo, E. M. Correlation of crack patterns and ring bands in spherulites of low molecular weight poly(1-lactic acid). Colloid. Polym. Sci. 2012, 290, 275-288.

58. Kan, T.; Isozaki, A.; Kanda, N.; Nemoto, N.; Konishi, K.; Takahashi, H.; Kuwata-

Gonokami, M.; Matsumoto, K.; Shimoyama, I. Enantiomeric switching of chiral metamaterial for terahertz polarization modulation employing vertically deformable MEMS spirals. Nat. Commun. 2015, 6, 8422 .

59. Kanda, N.; Konishi, K.; Kuwata-Gonokami, M. All-photoinduced terahertz optical activity. Opt. Lett. 2014, 39, 3274-3277.

60. Han, J.; You, J.; Li, X.; Duan, P.; Liu, M. Full-Color Tunable Circularly Polarized Luminescent Nanoassemblies of Achiral AIEgens in Confined Chiral Nanotubes. Adv. Mater. 2017, 29, 1606503.

61. Huo, S.; Duan, P.; Jiao, T.; Peng, Q.; Liu, M. Full- and White Color Circularly Polarized Luminescent Quantum Dots via Supramolecular Self-Assembly. Angew. Chem. Int. Ed. 2017, $56,12174-12178$.

62. Zhao, N.; Li, M.; Yan, Y.; Lam, J. W. Y.; Zhang, Y. L.; Zhao, Y. S.; Wong, K. S.; Tang, B. Z. A tetraphenylethene-substituted pyridinium salt with multiple functionalities: synthesis, stimuli-responsive emission, optical waveguide and specific mitochondrion imaging. J. Mater. Chem. C 2013, 1, 4640-4646.

63. Dolomanov, O. V.; Bourhis, L. J.; Gildea, R. J.; Howard, J. A. K.; Puschmann, H. OLEX2: a complete structure solution, refinement and analysis program. J. Appl. Cryst. 2009, $42,339-341$.

64. Zimmermann, T.; Rietdorf, J.; Pepperkok, R. Spectral imaging and its applications in live cell microscopy. FEBS Lett. 2003, 546, 87-92.

65. Sarasua, J.-R.; Prud'homme, R. E.; Wisniewski, M.; Le Borgne, A.; Spassky, N. Crystallization and Melting Behavior of Polylactides. Macromolecules 1998, 31, 3895-3905. 
66. Auriemma, F.; Alfonso, G. C.; De Rosa, C. Real-Time Fast Structuring of Polymers Using Synchrotron WAXS/SAXS Technique in Polymer Crystallization II: From Chain Microstructure to Processing; Auriemma, F.; Alfonso, G. C.; De Rosa, C., Eds.; Springer International Publishing: Cham, 2016; pp 127-165.

\section{Table of Contents}

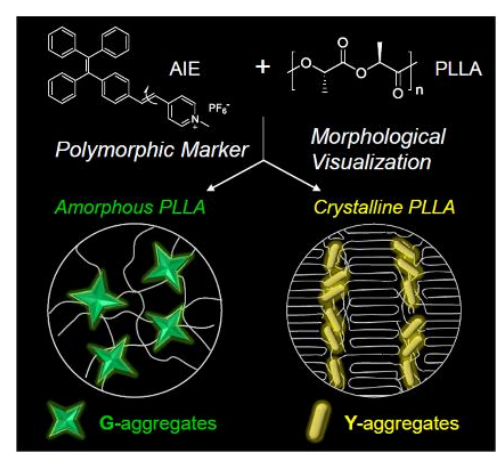




\section{Supporting Information}

Multiscale Morphological Visualization of a Semi-crystalline Polymer by a Polymorphic AIE Marker

Michidmaa Khorloo, ${ }^{\dagger, \perp}$ Yanhua Cheng, ${ }^{*},+\perp$ Haoke Zhang,${ }^{\dagger}$ Ming Chen,$^{\dagger}$ Herman H. Y. Sung, ${ }^{\dagger}$ Ian D. Williams, ${ }^{\dagger}$ Jacky W. Y. Lam ${ }^{\dagger}$ and Ben Zhong Tang $*, \dagger, \S$

This PDF file includes:

Tables S1 to S4

Figures $\mathbf{S 1}$ to $\mathbf{S 2 3}$

Table S1. The photophysical properties of polymorphic TPE-EP. Emission maximum wavelength $\left(\lambda_{\mathrm{em}}\right)$, fluorescence quantum yield $\left(\Phi_{\mathrm{F}}\right)$, average lifetime $(\tau)$, radiative rate constant $\left(k_{\mathrm{r}}\right)$ and non-radiative rate constant $\left(k_{n \mathrm{r}}\right)$.

\begin{tabular}{cccccc}
\hline Compound & $\begin{array}{c}\text { Fluorescence } \\
\lambda_{\mathrm{em}}(\mathrm{nm})\end{array}$ & $\Phi_{\mathrm{F}}^{\mathrm{a})}$ & $\tau(\mathrm{ns})^{\mathrm{b})}$ & $\begin{array}{c}k_{\mathrm{r}}^{\mathrm{c})} \\
\left(\times 10^{8} \mathrm{~s}^{-1}\right)\end{array}$ & $\begin{array}{c}\left.k_{\mathrm{nr}}{ }^{\mathrm{c}}\right) \\
\left(\times 10^{8} \mathrm{~s}^{-1}\right)\end{array}$ \\
\hline TPE-EP-G & 507 & 0.21 & 2.1 & 1.0 & 3.8 \\
TPE-EP-Y & 543 & 0.32 & 2.3 & 1.4 & 2.9 \\
TPE-EP-O & 575 & 0.23 & 2.4 & 1.0 & 3.2 \\
\hline measured by an integrating sphere.
\end{tabular}

b) Fluorescence lifetimes $(\tau)$ calculated according to equation of $\tau=\frac{\sum_{i} a_{i} \tau_{i}^{2}}{\sum_{i} a_{i} \tau_{i}}, \tau_{\mathrm{i}}$ is an individual lifetime and $a_{\mathrm{i}}$ is an amplitude of multi-exponential decay fit.

${ }^{c)}$ The radiative rate constant $k_{\mathrm{r}}=\Phi_{\mathrm{F}} / \tau$; the non-radiative rate constant $k_{n \mathrm{r}}=1 / \tau-k_{\mathrm{r}}$. 
Table S2. Crystal data and structure refinement for G- and Y-crystals.

\begin{tabular}{|c|c|c|}
\hline Crystal & TPE-EP-G & TPE-EP-Y \\
\hline Empirical formula & $\mathrm{C}_{69} \mathrm{H}_{58} \mathrm{Cl}_{2} \mathrm{~F}_{12} \mathrm{~N}_{2} \mathrm{P}_{2}$ & $\mathrm{C}_{34} \mathrm{H}_{28} \mathrm{~F}_{6} \mathrm{NP}$ \\
\hline Formula weight & 1276.01 & 595.54 \\
\hline Temperature, $\mathrm{K}$ & $100.03(11)$ & $100.01(10)$ \\
\hline Crystal system & monoclinic & monoclinic \\
\hline Space group & $\mathrm{P} 2{ }_{1} / \mathrm{n}$ & $\mathrm{P} 2{ }_{1} / \mathrm{c}$ \\
\hline$a, \AA$ & $11.2525(2)$ & $24.8939(7)$ \\
\hline$b, \AA$ & $9.21940(13)$ & $9.1783(2)$ \\
\hline$c, \AA$ & $59.1397(9)$ & $12.5794(3)$ \\
\hline$\alpha,{ }^{\circ}$ & 90 & 90 \\
\hline$\beta, \circ$ & $93.8053(14)$ & $98.168(3)$ \\
\hline$\gamma, \circ$ & 90 & 90 \\
\hline Volume, $\AA^{3}$ & $6121.68(17)$ & $2845.01(13)$ \\
\hline$Z$ & 4 & 4 \\
\hline$\rho_{\text {calc }}, \mathrm{g} / \mathrm{cm}^{3}$ & 1.384 & 1.390 \\
\hline$\mu, \mathrm{mm}^{-1}$ & 2.143 & 1.422 \\
\hline$F(000)$ & 2632.0 & 1232.0 \\
\hline Crystal size, $\mathrm{mm}^{3}$ & $0.2 \times 0.02 \times 0.02$ & $0.2 \times 0.08 \times 0.02$ \\
\hline Radiation & $\mathrm{CuK} \alpha(\lambda=1.54184)$ & $\mathrm{CuK} \alpha(\lambda=1.54184)$ \\
\hline $2 \theta$ range for data collection, ${ }^{\circ}$ & 7.918 to 134.996 & 10.772 to 134.998 \\
\hline Index ranges & $\begin{array}{l}-13 \leq \mathrm{h} \leq 13,-11 \leq \mathrm{k} \leq 10 \\
-35 \leq 1 \leq 70\end{array}$ & $\begin{array}{l}-29 \leq \mathrm{h} \leq 28,-10 \leq \mathrm{k} \leq 10 \\
-15 \leq 1 \leq 13\end{array}$ \\
\hline Reflections collected & 32777 & 14173 \\
\hline Independent reflections & $\begin{array}{l}10976\left[\mathrm{R}_{\text {int }}=0.0416,\right. \\
\left.\mathrm{R}_{\text {sigma }}=0.0423\right]\end{array}$ & $\begin{array}{l}4687\left[R_{\text {int }}=0.0309,\right. \\
\left.R_{\text {sigma }}=0.0300\right]\end{array}$ \\
\hline Data/restraints/parameters & $10976 / 86 / 849$ & $4687 / 6 / 389$ \\
\hline Goodness-of-fit on $\mathrm{F}^{2}$ & 1.036 & 1.019 \\
\hline Final $\mathrm{R}$ indexes $[\mathrm{I}>=2 \sigma(\mathrm{I})]$ & $\begin{array}{l}\mathrm{R}_{1}=0.0623, \mathrm{wR}_{2}= \\
0.1501\end{array}$ & $\mathrm{R}_{1}=0.0869, \mathrm{wR}_{2}=0.2128$ \\
\hline Final $\mathrm{R}$ indexes [all data] & $\begin{array}{l}\mathrm{R}_{1}=0.0744, \mathrm{wR}_{2}= \\
0.1577\end{array}$ & $\mathrm{R}_{1}=0.0932, \mathrm{wR}_{2}=0.2163$ \\
\hline Largest diff. peak/hole, e. $\AA^{-3}$ & $0.95 /-0.71$ & $0.95 /-0.43$ \\
\hline
\end{tabular}



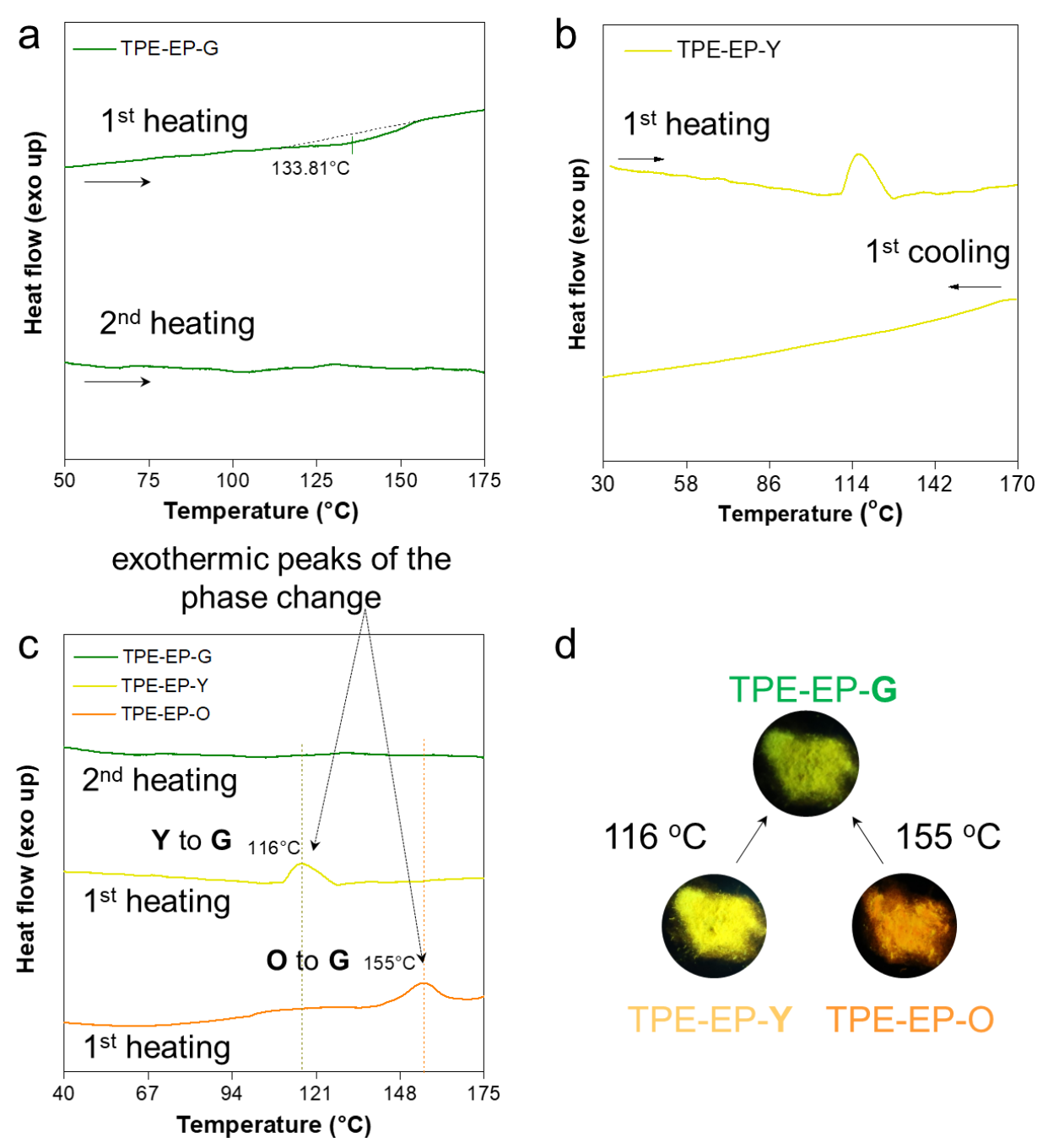

Figure S1. DSC analysis of TPE-EP polymorphs and their fluorescent photographs. (a) DSC traces of G-crystals at first (upper) and second heating (bottom) processes. (b) DSC traces of $\mathbf{Y}$-crystals at first heating (upper) and cooling (bottom) processes. (c) DSC traces of G-, Y-and O-crystals with the phase-transition temperatures. (d) Heat-triggered phase transformation. The luminescent color changes of $\mathbf{Y}$ to $\mathbf{G}$, and $\mathbf{O}$ to $\mathbf{G}$ were initiated at $116{ }^{\circ} \mathrm{C}$ and $155^{\circ} \mathrm{C}$, respectively.

In Figure S1a, the first heating scan of G-crystals revealed a broad endothermic peak at $\sim 134{ }^{\circ} \mathrm{C}$, indicating the evaporation of included solvent in crystalline lattice. The disappearance of the broad peak at the second heat scan further demonstrates the exclusion of the solvent molecules. In Figure S1b, a thermal phase transformation from $\mathbf{Y}$ to $\mathbf{G}$ was initiated at $116^{\circ} \mathrm{C}$. 
No reverse phase was observed when the sample was cooled. In Figure S1c, a similar thermal phase transformation from $\mathbf{O}$ to $\mathbf{G}$ was observed at $155^{\circ} \mathrm{C}$. The DSC analyses indicate that $\mathbf{Y}$ crystals and $\mathbf{O}$-crystals are metastable while $\mathbf{G}$-crystals are thermodynamically stable. The luminescent color changes of TPE-EP polymorphs were shown in Figure S1d.

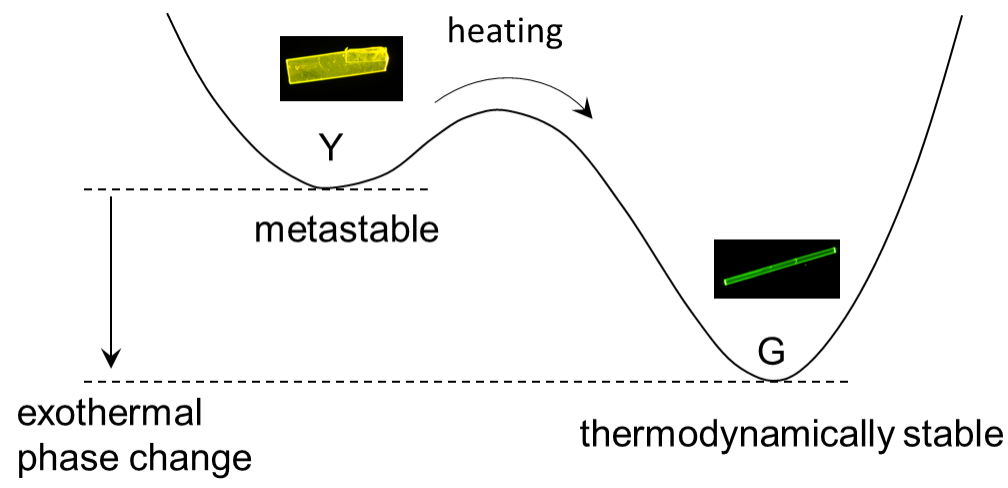

Figure S2. Diagram of the thermodynamic energies for G- and Y-crystals.
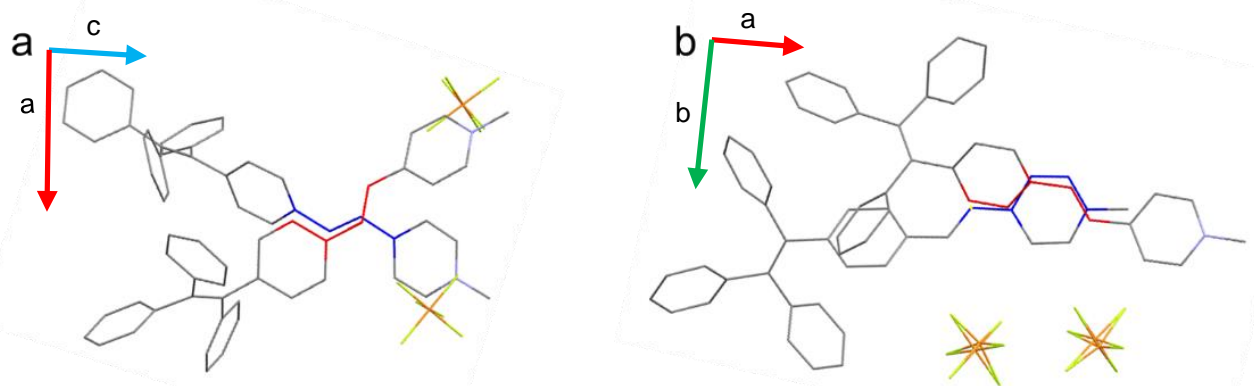

Figure S3. Molecular overlap of the (a) G-crystals and (b) Y-crystals. 


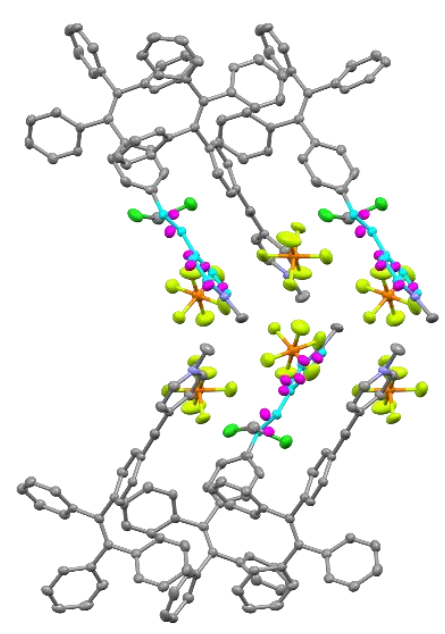

Figure S4. Ellipsoid drawing of the crystal structure of $\mathbf{G}$ with crystallographically ordered units (G1) and crystallographically disordered units (G2) with two conformations. Conformers I and II are colored with blue and pink, respectively. The hydrogen atoms in G-crystals are omitted for clarity.
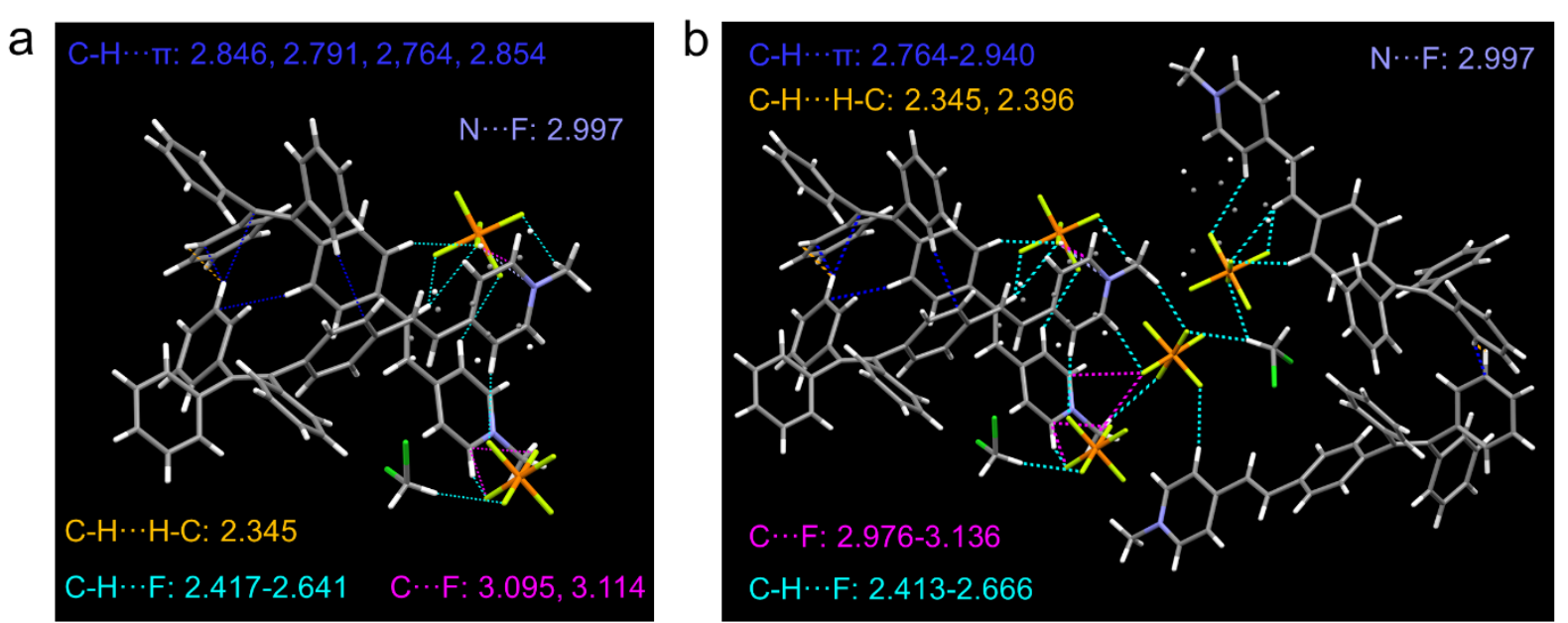

Figure S5. Multiple interactions in G-crystals. (a) Multiple intermolecular interactions between $\mathbf{G 1}$ and G2. (b) Intermolecular packing viewed along the $b$ axis. 


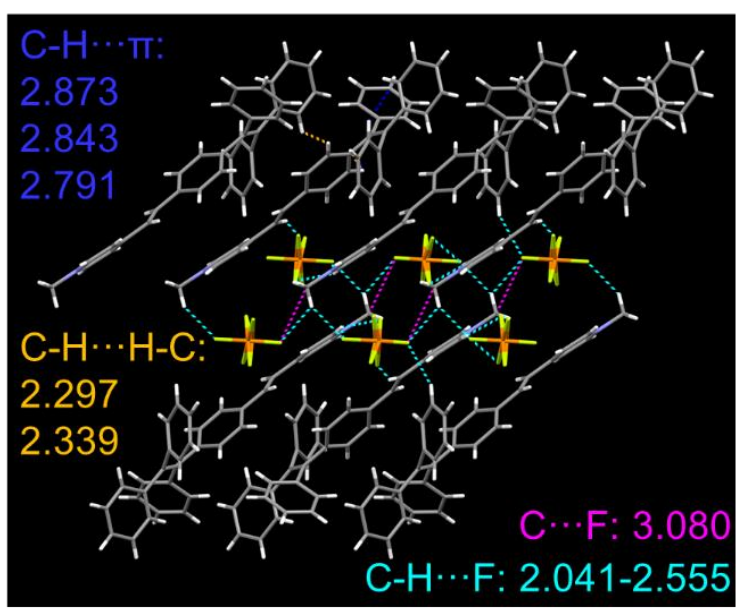

Figure S6. Multiple interactions in Y-crystals. Short contacts in Y-crystals and intermolecular packing viewed along the $b$ axis.

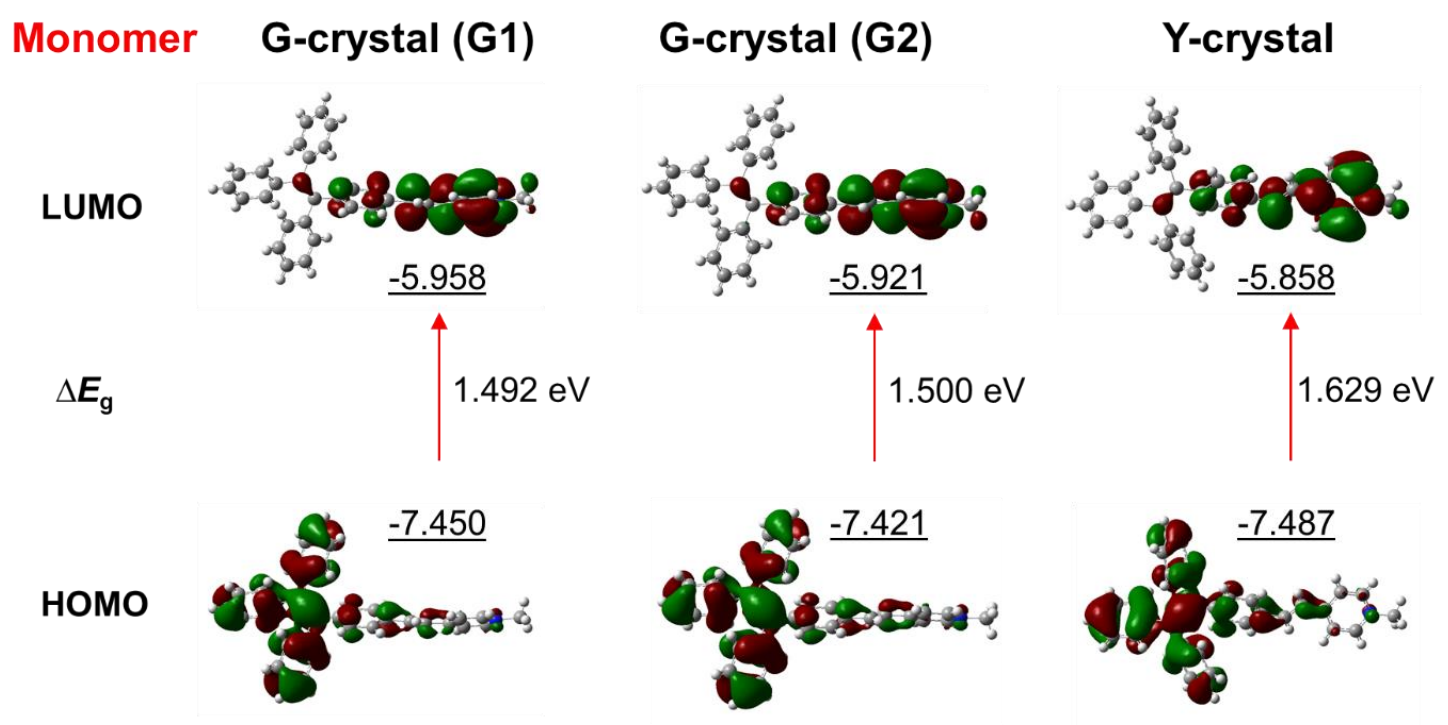

Figure S7. Calculated HOMO and LUMO levels for TPE-EP monomer selected from single crystals. The calculation is obtained from B3LYP/6-311G(d,p) basis set by Gaussian 09 program. 

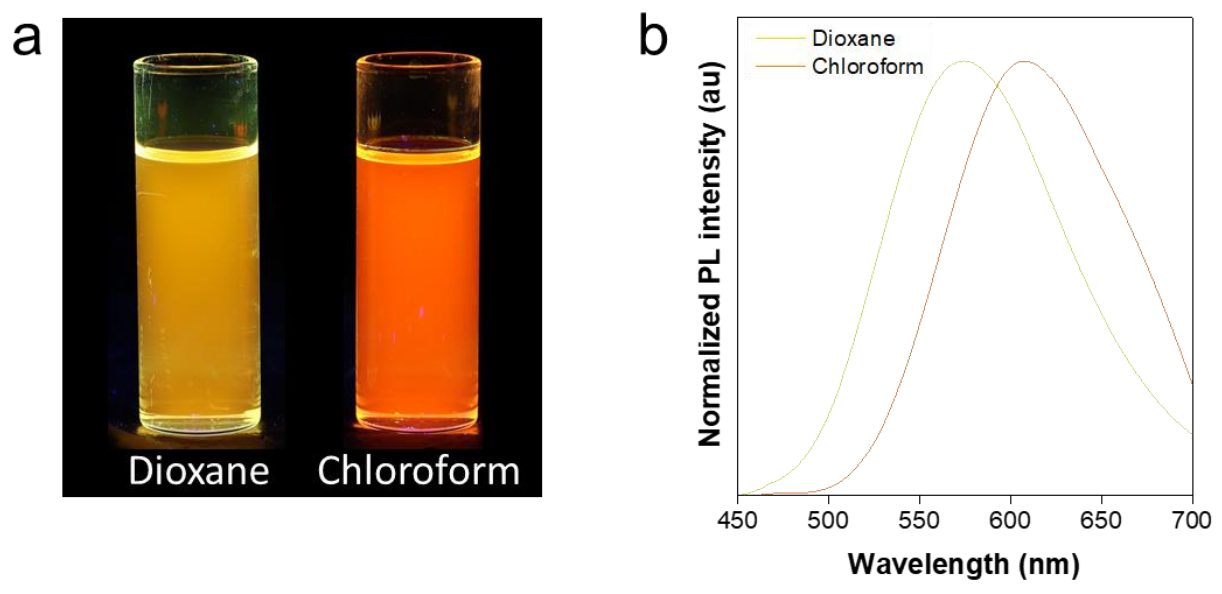

Figure S8. Solvatochromic properties of TPE-EP. (a) Photographs of the solutions of TPEEP in organic solvents of dioxane and chloroform taken under UV light. Excitation wavelength: $365 \mathrm{~nm}$. (b) Normalized fluorescence spectra of TPE-EP in dioxane (yellow line) and chloroform (orange line).
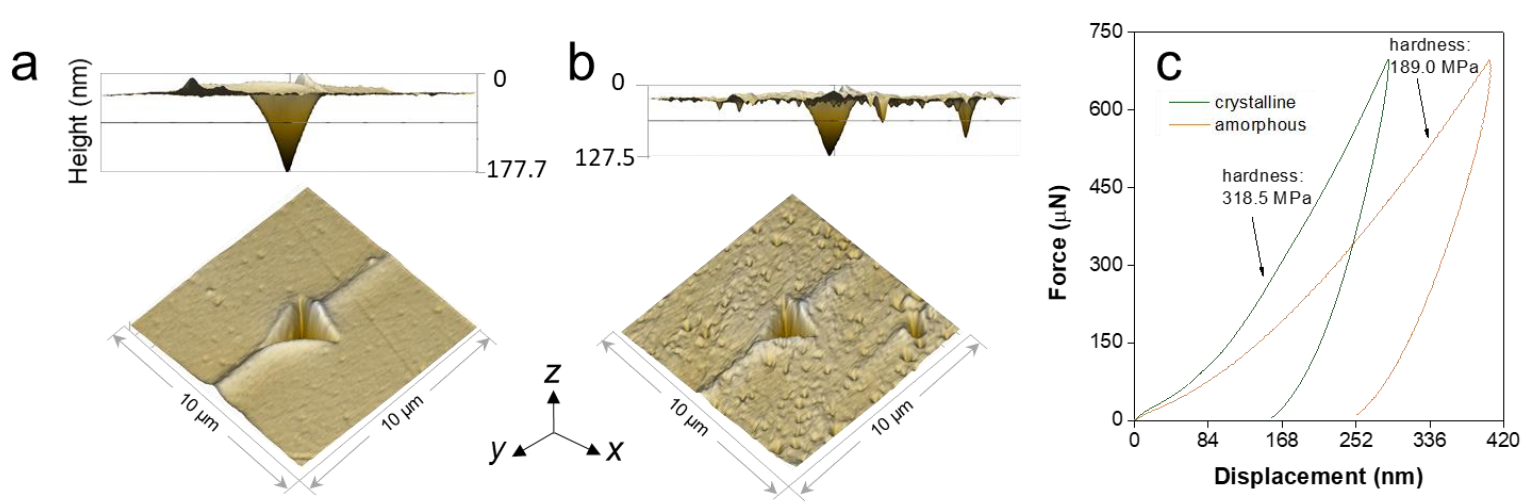

Figure S9. Mechanical properties of amorphous and crystalline PLLA studied by nanoindentation test. Nanoindentation images of (a) amorphous and (b) crystalline PLLA films. (c) Force-displacement curves of the amorphous (green line) and crystalline (yellow line) PLLA films. 

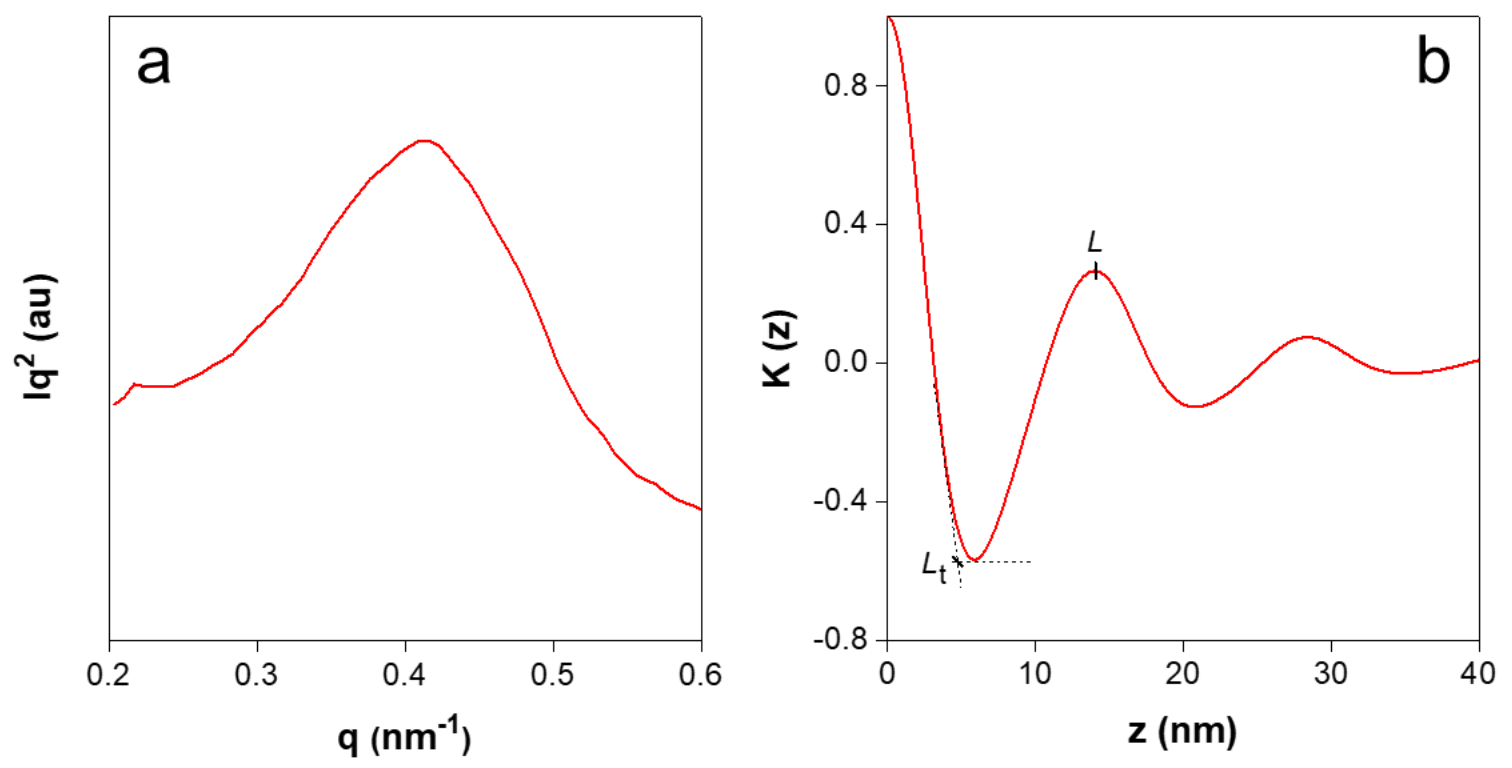

Figure S10. (a) Lorentz-corrected SAXS pattern of TPE-EP-labelled crystalline PLLA and (b) corresponding 1D correlation function profile. The long period $(L=14 \mathrm{~nm})$ of crystalline PLLA was determined by the position of first peak, while the average thickness of the thinner layers $\left(L_{\mathrm{t}}=5 \mathrm{~nm}\right)$ was calculated by the connection between tangent and baseline of the first wave trough. ${ }^{1}$ Considering the crystallinity $\left(\chi_{c}=48 \%\right)$, the thinner layers were identified as amorphous phase that sandwiched between two crystalline lamellas. 

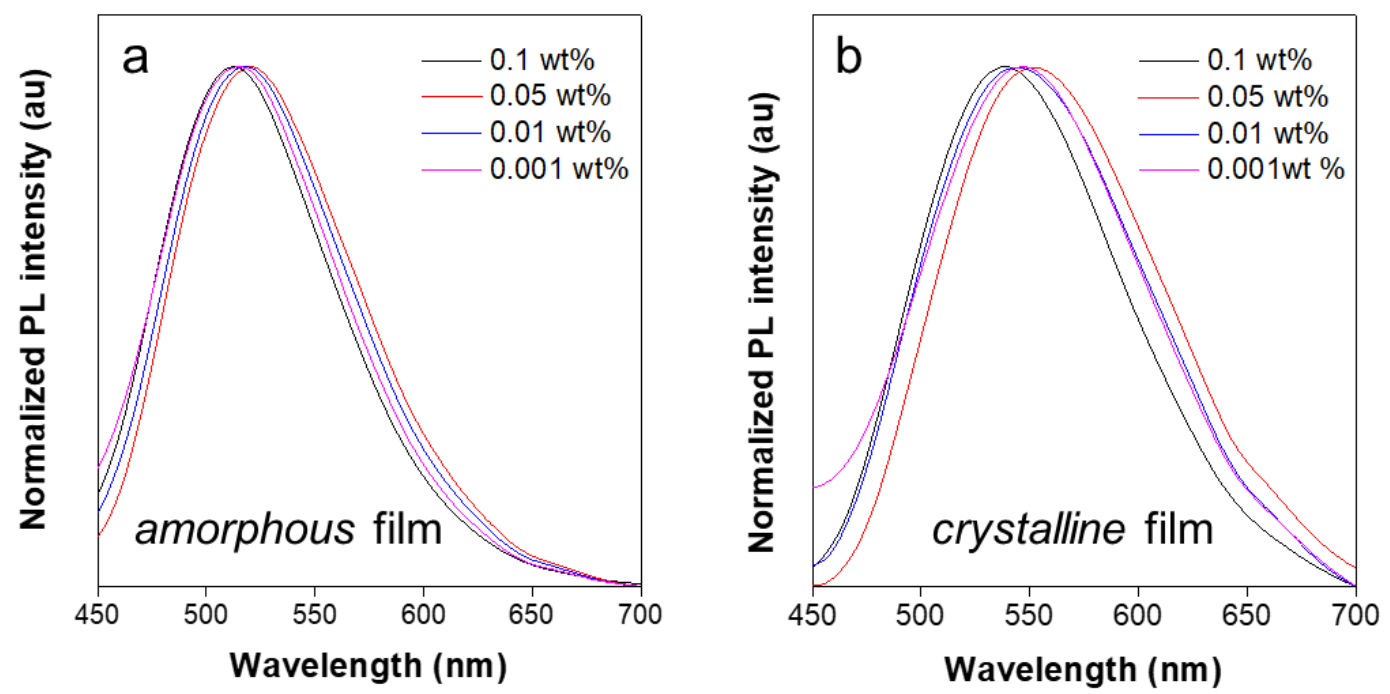

Figure S11. Normalized PL spectra of TPE-EP-labeled (a) amorphous and (b) crystalline PLLA films with various doping ratio.

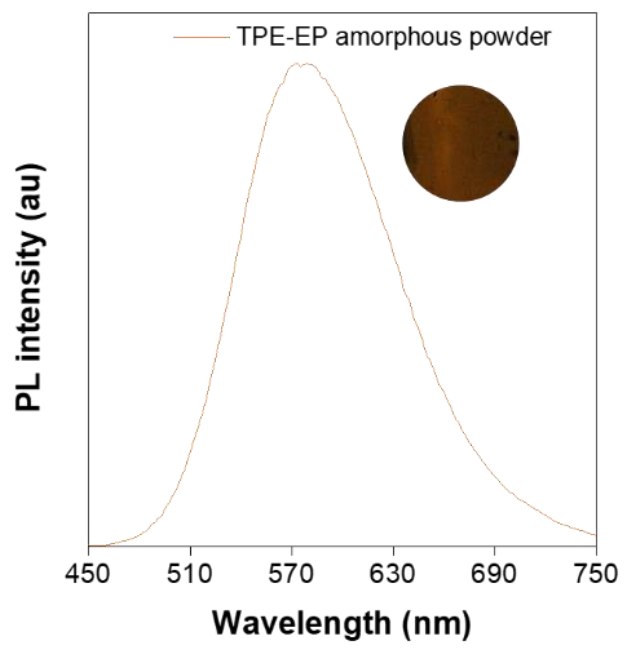

Figure S12. The PL spectrum of TPE-EP amorphous powder. Inset: image of amorphous TPEEP powder under $365 \mathrm{~nm}$ UV light. The amorphous powder of TPE-EP shows orange emission. 
a
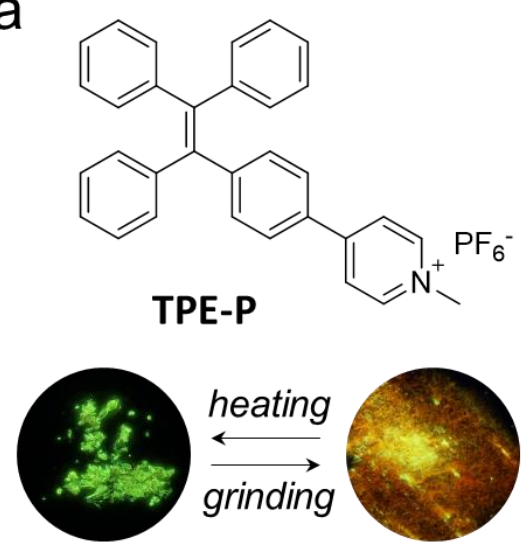

C

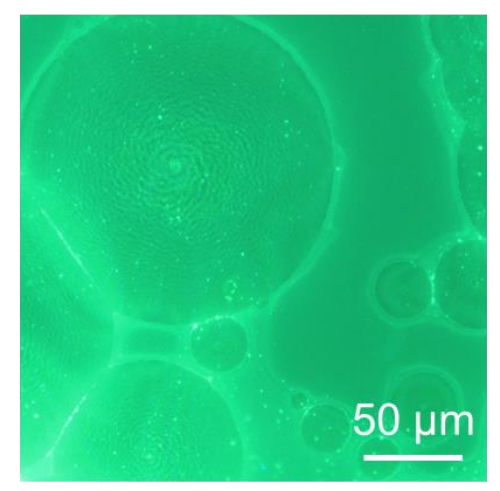

b

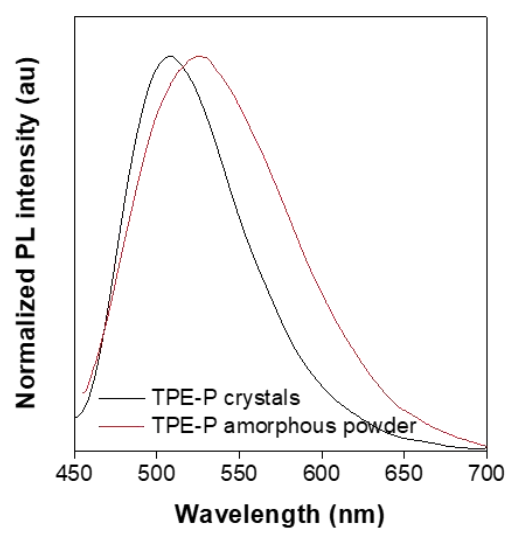

d

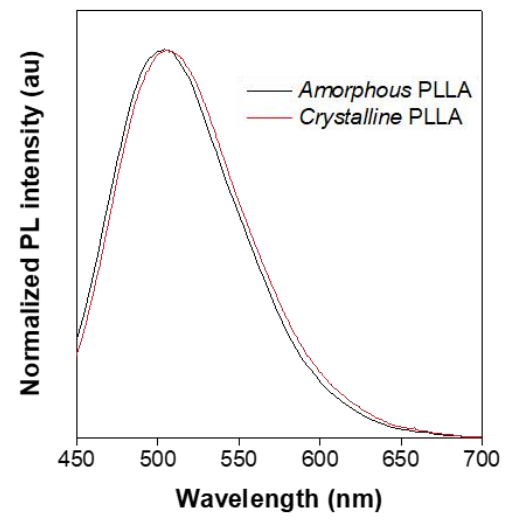

Figure S13. Control demonstration by TPE-P. (a) (top) Chemical structure and (bottom) fluorescent images of TPE-P taken under $365 \mathrm{~nm}$ UV light. Bottom left: green emissive crystal, bottom right: orange amorphous powder. (b) Respective PL spectra of TPE-P crystals and TPEP amorphous powder. (c) Fluorescence micrograph of the TPE-P-labelled PLLA film with a mixture of crystalline and amorphous regions. (d) Normalized PL spectra of TPE-P-labelled PLLA films at different phases.

In order to further prove the nanocrystalline states of TPE-EP in PLLA, we use another AIE molecule showing similar chemical structure with that of TPE-EP, namely TPE-P. In TPE$\mathrm{P}$, the pyridinium moiety is directly attached to TPE unit (Figure S13a). For TPE-P molecules, only crystalline crystals and amorphous powders are observed, which show green $\left(\lambda_{\mathrm{em}}=508\right.$ $\mathrm{nm})$ and yellow $\left(\lambda_{\mathrm{em}}=526 \mathrm{~nm}\right)$ emission, respectively (Figure S13b). Experiments were performed to fabricate polymer films that embedded with TPE-P, similar to that for TPE-EP- 
labelled PLLA films. In Figure S13c and d, both amorphous and crystalline PLLA phases that labelled with TPE-P show green emission $\left(\lambda_{\mathrm{em}}=\sim 506 \mathrm{~nm}\right)$. The emission maximum is very close to that of TPE-P crystals. The results further demonstrate that segregated AIE molecules are in forms of nanocrystals rather than amorphous aggregates in PLLA.

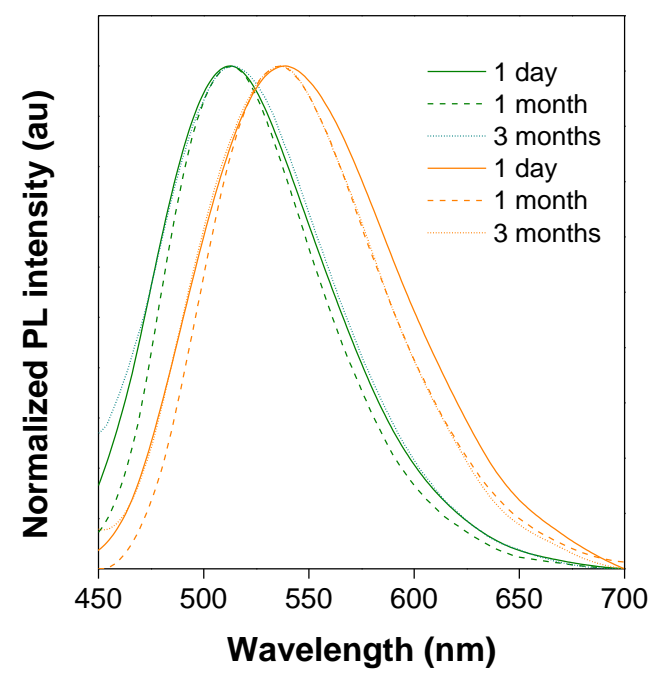

Figure S14. PL spectra of TPE-EP-labelled PLLA films monitored during three months. Green lines: amorphous PLLA films embedded with TPE-EP; Orange lines: crystalline PLLA films embedded with TPE-EP.

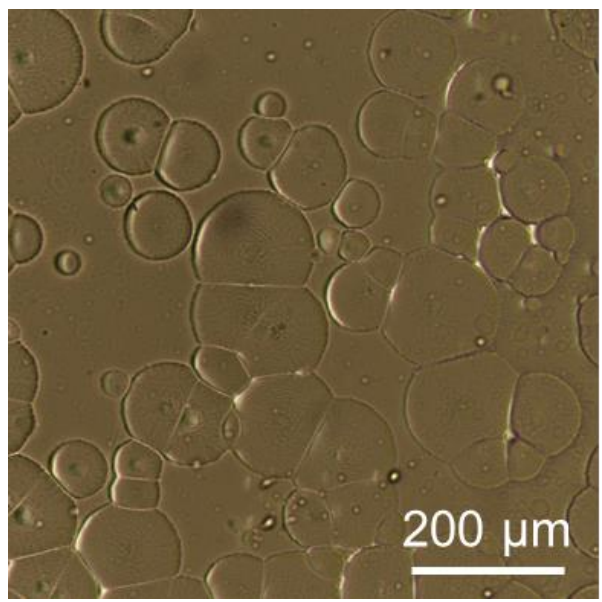

Figure S15. Microscopic image of the PLLA films taken under bright filed. 

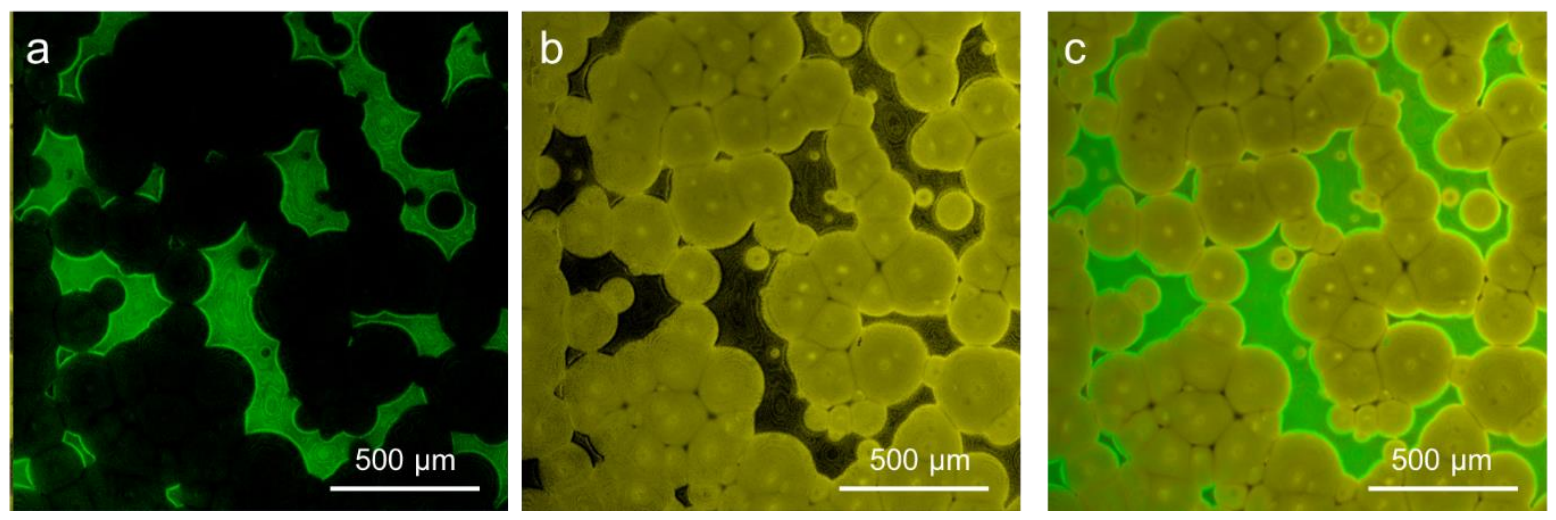

Figure S16. Linearly unmixed CFM images of TPE-EP-labelled PLLA with a mixture of crystalline and amorphous regions. (a) Amorphous PLLA marked by TPE-EP-G shown as green and (b) crystalline PLLA marked by TPE-EP-Y shown as yellow in confocal image by linear unmixing two-channel imaging. Channel one of green was selected between $410-516 \mathrm{~nm}$ and Channel two of yellow was selected between 518-688 nm. (c) Merged images of panels a and $\mathbf{b}$.
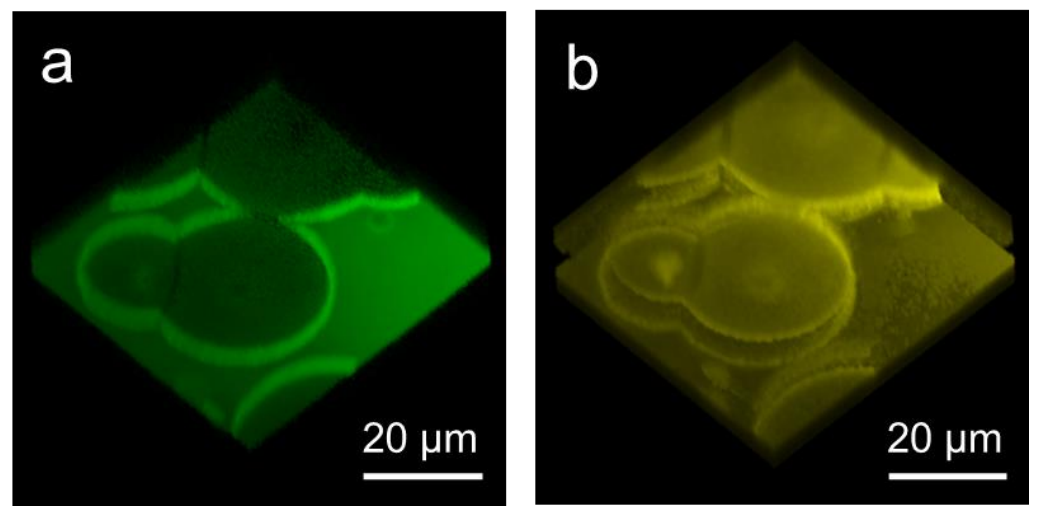

Figure S17. Confocal fluorescent 3D images of crystalline spherulites under $405 \mathrm{~nm}$ laser irradiation. (a) Amorphous PLLA marked by TPE-EP-G shown as green and (b) crystalline PLLA marked by TPE-EP-Y shown as yellow in confocal image using linear unmixing twochannel Z-scanning. Channel one was selected between 410-516 nm and Channel two was selected between 518-688 $\mathrm{nm}$. 

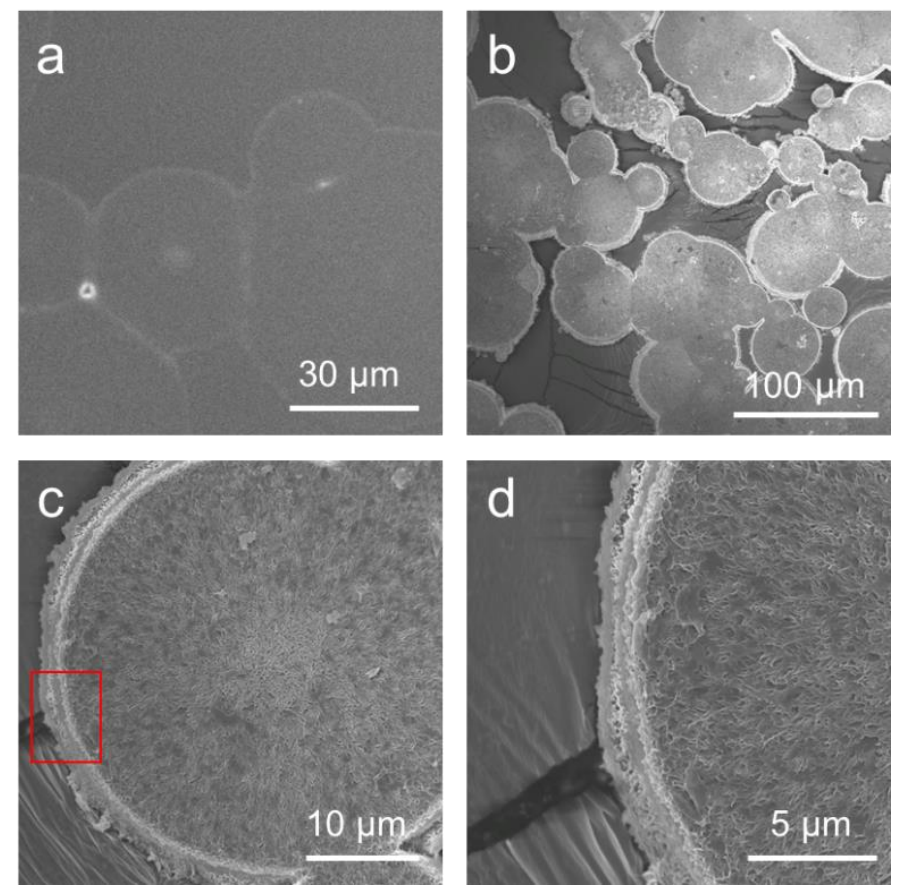

Figure S18. SEM image of PLLA film with a mixture of amorphous and crystalline regions (a) before and (b, c, d) after acetone etching. (c) SEM image of the crystalline spherulites and (d) magnified image of the selected area in c.

After gold sputtering, the morphology of the film is presented as shown in Figure S18a. In contrast, only ambiguous polymer crystalline shape can be observed in the SEM image. It has been reported that the density of amorphous PLLA $\left(1.248 \mathrm{~g} \mathrm{ml}^{-1}\right)$ is less than that of crystalline states $\left(1.290 \mathrm{~g} \mathrm{ml}^{-1}\right){ }^{2}$ Acetone could dissolve the amorphous PLLA rather than the crystalline region. As a result, SEM images of 3D spherulites shown in Figure S18b, c and d were created. 

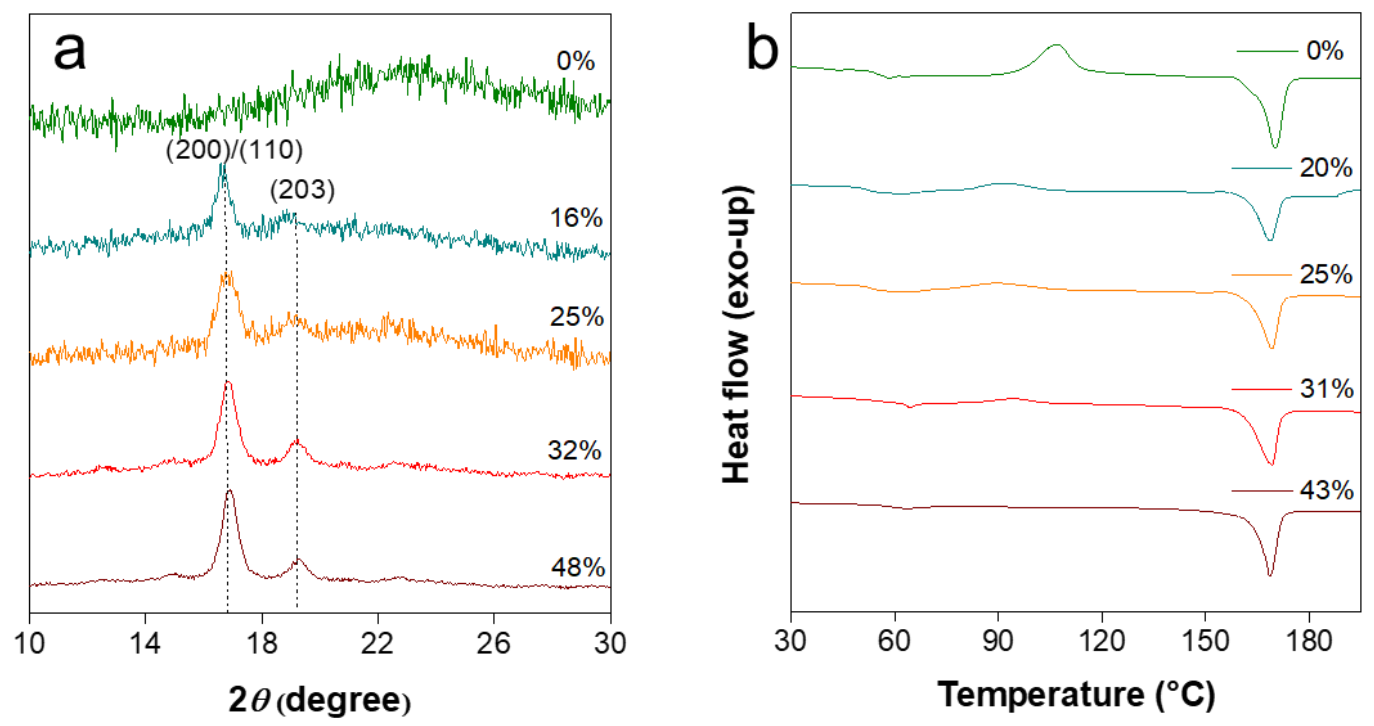

Figure S19. Crystallinity measurement by WAXD and DSC. (a) WAXD profiles of TPEEP-labeled PLLA at different polymer crystallinity. (b) DSC thermograms of TPE-EP-labelled PLLA at different crystallinity, which were recorded under nitrogen at the first heating scan at a rate of $10{ }^{\circ} \mathrm{C} \mathrm{min}^{-1}$. Corresponding melting enthalpy values, exothermal enthalpy values, crystallization temperatures, melting temperatures, and crystallinity values are listed in Table S3.

Table S3. Thermal profiles of PLLA with varied crystallinity.

\begin{tabular}{ccccc}
\hline $\begin{array}{c}\mathbf{T}_{\mathbf{c}} \\
\left({ }^{\circ} \mathrm{C}\right)\end{array}$ & $\begin{array}{c}\Delta \mathbf{H}_{\mathbf{c}} \\
\left(\mathrm{J} \mathrm{g}^{-1}\right)\end{array}$ & $\begin{array}{c}\mathbf{T}_{\mathbf{m}} \\
\left({ }^{\circ} \mathrm{C}\right)\end{array}$ & $\begin{array}{c}\Delta \mathbf{H}_{\mathbf{m}} \\
\left(\mathrm{J} \mathrm{g}^{-1}\right)\end{array}$ & $\begin{array}{c}\boldsymbol{\chi}_{\mathbf{c}} \\
(\%)\end{array}$ \\
\hline 107.0 & -37.52 & 170.1 & 37.28 & $\mathbf{0}$ \\
92.02 & -12.65 & 162.8 & 34.05 & $\mathbf{2 0 . 2}$ \\
89.46 & -10.37 & 163.3 & 36.52 & $\mathbf{2 4 . 7}$ \\
94.14 & -6.551 & 161.4 & 39.58 & $\mathbf{3 1 . 6}$ \\
$/$ & $/$ & 165.1 & 45.80 & $\mathbf{4 3 . 2}$ \\
\hline
\end{tabular}




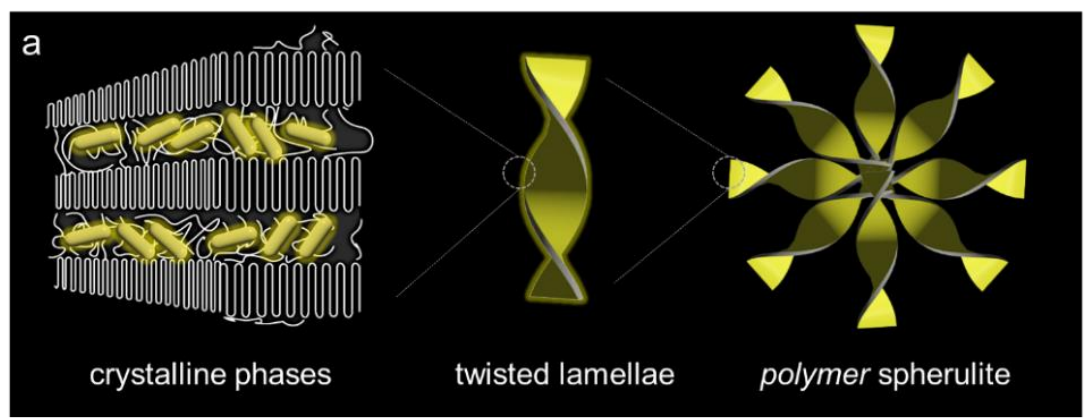

b

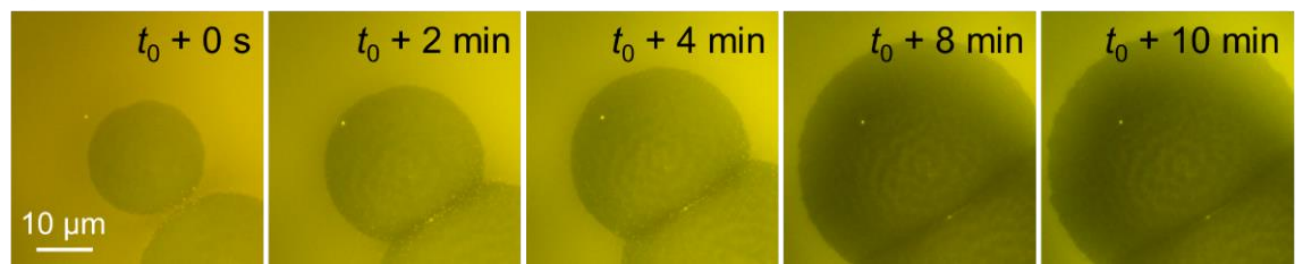

Figure S20. (a) Illustration of the formation of spiral-banded spherulite that labeled with TPEEP. ${ }^{1}$ TPE-EP molecules in forms of nanocrystalline $\mathbf{Y}$ are distributed in between two PLLA lamellas. (b) Real-time fluorescent photos of the evolution process of one period (10 $\mathrm{min})$ in a PLLA spherulite.

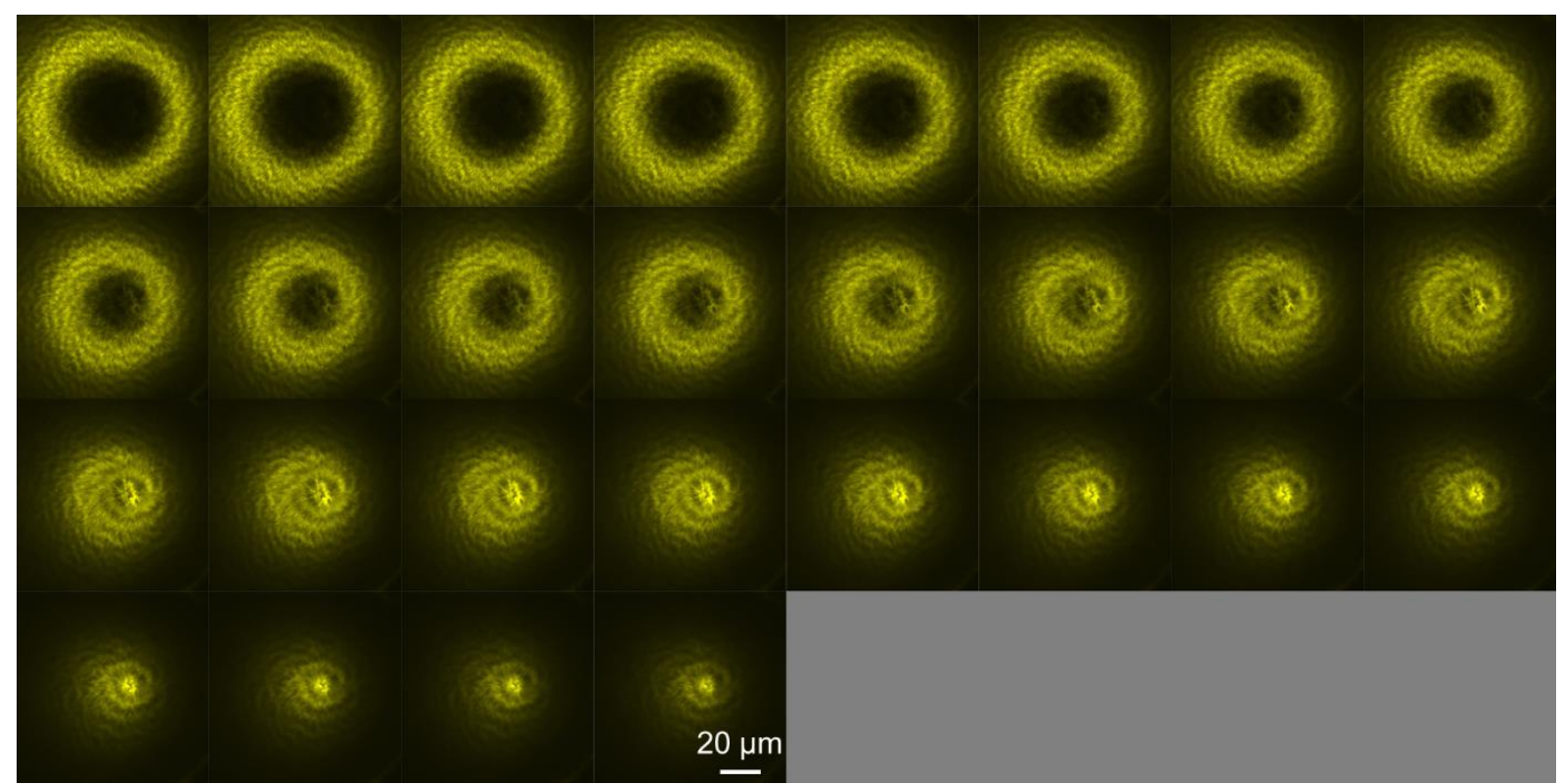

Figure S21. Confocal images of each slice of spiral banded spherulites labelled with TPE-EP using Z-scan technique. Excitation wavelength: $405 \mathrm{~nm}$. The optical signal was selected between 400-700 nm. The images clearly show the Archimedean spiral structure of PLLA 
spherulites. The 3D spiral highlighted with bright-yellow is presented in the upward vertical direction.

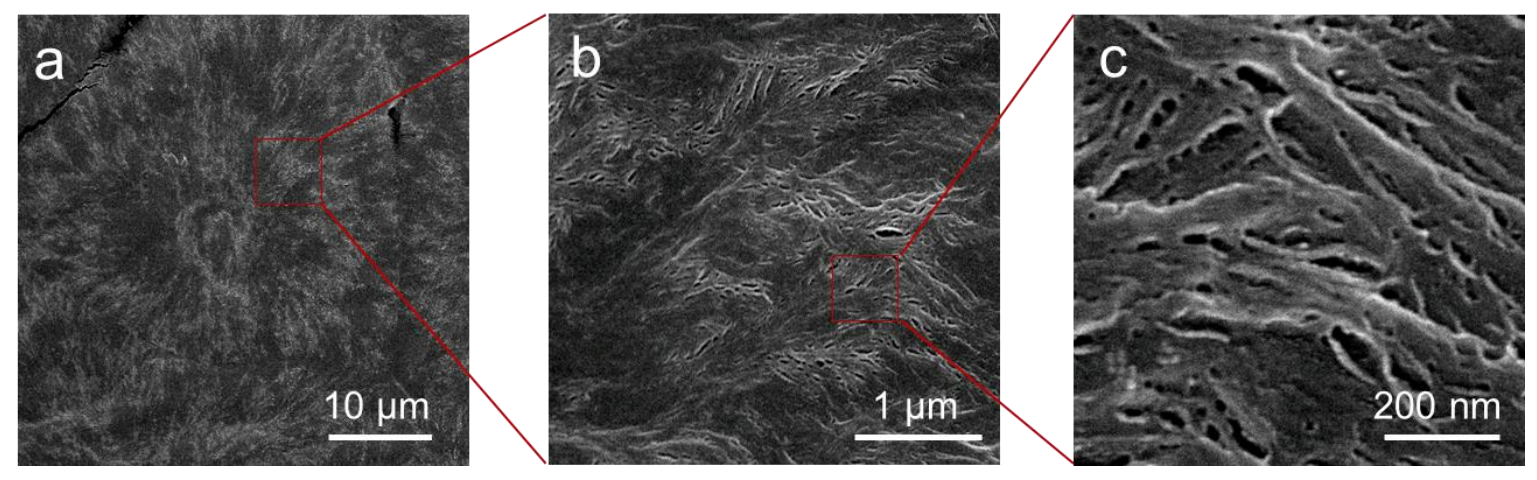

Figure S22. SEM images of (a) solvent etched crystalline spherulite and (b, c) magnified images of the respective marked area.

Table S4. The measurement conditions of CPL measurements on CPL-300 instrument.

\begin{tabular}{cc}
\hline \multicolumn{2}{c}{ CPL Measurement Conditions } \\
\hline Excitation Wavelength & $340 \mathrm{~nm}$ \\
Excitation Slit Width & $3 \mathrm{~mm}$ \\
Data Pitch & $0.1 \mathrm{~nm}$ \\
Measure Range & $660-360 \mathrm{~nm}$ \\
Emission Slit Width & $3 \mathrm{~mm}$ \\
Scan Speed & $50 \mathrm{~nm} / \mathrm{min}$ \\
D.I.T. & $8 \mathrm{sec}$ \\
\hline
\end{tabular}



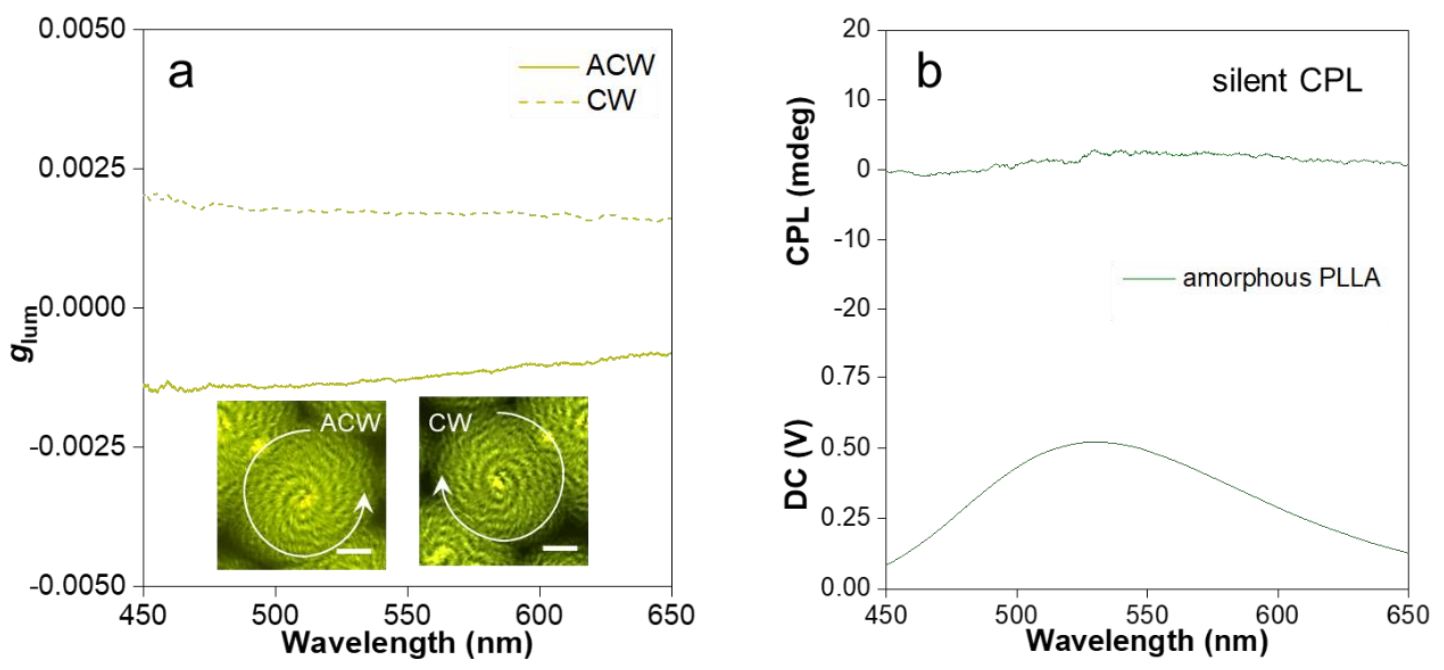

Figure S23. (a) CPL dissymmetry factor $g_{\text {lum }}$ as a function of the wavelength. The spectra were recorded on the TPE-EP-labelled crystalline film that excited from two different sides. Solid line: Excited from the side that the spiral turn direction was anti-clockwise (ACW). Dash line: Excited from the side that the spiral turn direction was clockwise $(\mathrm{CW})$. The spiral morphology of polymer spherulites observed from different sides are shown in the insets. Scale bars are 20 $\mu \mathrm{m}$. (b) CPL spectrum of amorphous PLLA film that labelled with TPE-EP, which show silent signal. The results indicate that the CPL response is originated from the superstructure of the spiral patterns.

\section{References}

1. Li, M.-C.; Wang, H.-F.; Chiang, C.-H.; Lee, Y.-D.; Ho, R.-M. Lamellar-Twisting-Induced Circular Dichroism of Chromophore Moieties in Banded Spherulites with Evolution of Homochirality. Angew. Chem. Int. Ed. 2014, 53, 4450-4455.

2. Madhavan Nampoothiri, K.; Nair, N. R.; John, R. P. An overview of the recent developments in polylactide (PLA) research. Bioresour. Technol. 2010, 101, 8493-8501. 
